Merci d'utiliser le titre suivant lorsque vous citez ce document :

Geloso Grosso, M. (2004-07-22), « Organisation des negociations fondées sur le principe des "offres et requêtes" dans le cadre de l'AGCS : Le cas des services juridiques ", Éditions OCDE, Paris.

http://dx.doi.org/10.1787/804406835842

\title{
Organisation des
}

negociations fondées sur le principe des "offres et requêtes" dans le cadre de I'AGCS

\author{
LE CAS DES SERVICES JURIDIQUES
}

Massimo Geloso Grosso

La version originale de ce document a été publiée comme suit :

Geloso Grosso, M. (2004-06-14), "Managing Request-Offer Negotiations Under the GATS: The Case of Legal Services", OECD Trade Policy Papers, No. 2, OECD Publishing, Paris. http://dx.doi.org/10.1787/828686816540 
Organisation de Coopération et de Développement Economiques

Organisation for Economic Co-operation and Development

22-Jul-2004

DIRECTION DES ECHANGES

Français - Or. Anglais

COMITE DES ECHANGES

Groupe de travail du Comité des échanges

ORGANISATION DES NÉGOCIATIONS FONDÉES SUR LE PRINCIPE DES "OFFRES ET REQUETES" DANS LE CADRE DE L'AGCS : LE CAS DES SERVICES JURIDIQUES

Document de travail $\mathrm{N}^{\circ} \mathbf{2}$ de l'OCDE sur les politiques commerciales

par Massimo Geloso Grosso

Tous les documents de travail de la Direction des échanges sont désormais disponibles sur le site Web de l'OCDE http://www.oecd.org/trade 


\section{RESUME}

La présente étude fait partie du projet sur les échanges de services que l'OCDE mène en coopération avec la CNUCED en vue d'aider les Membres de l'OMC à organiser les négociations fondées sur le principe des «offres et requêtes » dans le cadre de l'AGCS. Elle a pour principal objectif de permettre aux responsables des Membres de l'OMC de mieux connaître les questions qui revêtent une importance particulière pour le secteur des services juridiques, ainsi que de savoir comment les aborder au cours des négociations. Si le Cycle d'Uruguay n'a permis d'obtenir qu'une libéralisation limitée des échanges de services juridiques, les négociations en cours offrent la possibilité de la faire progresser davantage, avec - à la clef - d'importants avantages économiques pour tous les pays. En outre, la caractéristique propre aux services juridiques est que les inconvénients pouvant découler de la libéralisation - tant en termes d'incapacité du marché à atteindre les objectifs sociaux que du point de vue du remplacement des fournisseurs locaux - seront vraisemblablement moindres dans ce secteur des services.

Mots clés : juridiques, services, obstacles, avantages, exportations, libéralisation, réglementation.

\section{REMERCIEMENTS}

La présente étude a été rédigée par Massimo Geloso Grosso de la Direction des échanges de l'OCDE. Elle a bénéficié d'importantes contributions de Maxine Kennett et a été supervisée par Julia Nielson et Dale Andrew, chef de la Division des liens en matière de politique commerciale. Elle a été examinée par le Groupe de travail du Comité des échanges, qui a décidé de diffuser plus largement ces conclusions en déclassifiant l'étude sous la responsabilité du Secrétaire général. On pourra en obtenir le texte en français et en anglais sur le site Web de l'OCDE : http://www.oecd.org/trade.

L'auteur tient à exprimer sa gratitude à Patrick Bourke, Markus Jelitto, Dale Honeck, Alison Hook et Carlos Gimeno Verdejo pour l'utilité des remarques et observations qu'ils lui ont adressées durant la préparation de l'étude.

Droits d'auteur : OCDE 2004

Les demandes de reproduction ou de traduction totales ou partielles de cette publication doivent être adressées à :

M. le Chef du Services des Publications, OCDE

2, rue André-Pascal, 75775 PARIS CEDEX 16, France 


\section{TABLE DES MATIÈRES}

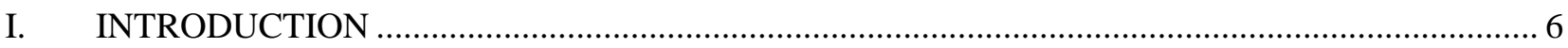

II. CROISSANCE DU SECTEUR DES SERVICES JURIDIQUES ….............................................. 7

III. COURANTS D'ECHANGES DE SERVICES JURIDIQUES ET MESURES RESTREIGNANT

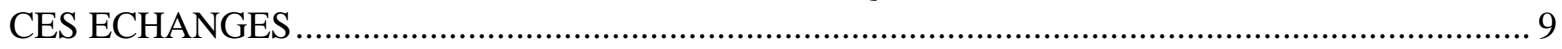

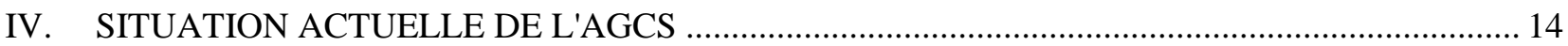

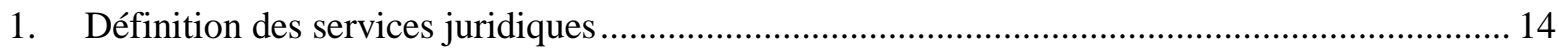

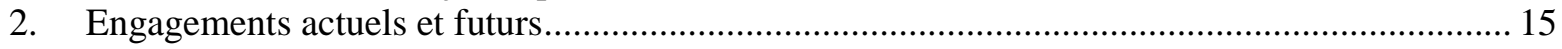

V. AVANTAGES ESCOMPTES, PROBLÈMES À RESOUDRE ET EFFORTS DE RÉFORME DE

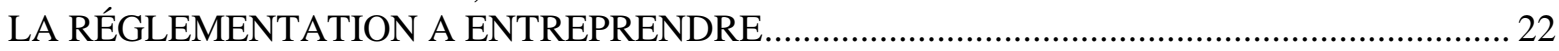

1. Avantages potentiels de l'ouverture des marchés pour les services juridiques........................... 22

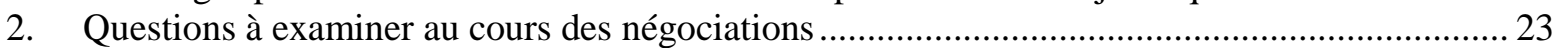

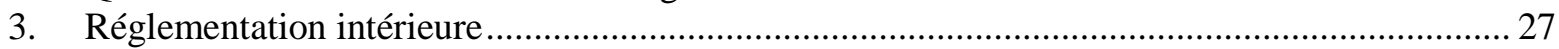

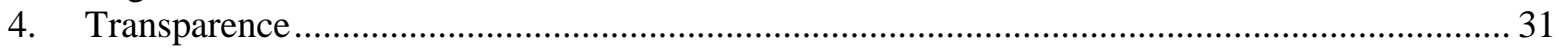

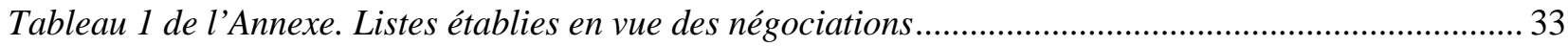

Tableau 2 de l'Annexe. Principaux cabinets de consultations juridiques du monde en 2002 ...................... 36

Tableau 3 de l'Annexe. Exemption de l'application de la clause NPF dans le secteur des services

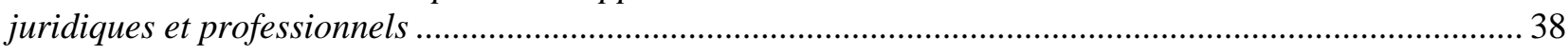

Tableau 4 de l'Annexe. Engagements relatifs aux services juridiques contractes dans le cadre du cycle d'Uruguay..... 


\title{
ORGANISATION DES NÉGOCIATIONS FONDÉES SUR LE PRINCIPE DES "OFFRES ET REQUÊTES" DANS LE CADRE DE L'AGCS : LE CAS DES SERVICES JURIDIQUES
}

\author{
PRINCIPALES CONCLUSIONS
}

Le présent document s'inscrit dans le cadre du projet en cours sur les échanges de services entrepris en vue d'établir un ensemble de listes sectorielles, en coopération avec la CNUCED, en partant des listes de négociation génériques mises au point dans la partie II du document "Organisation des négociations fondées sur le principe des 'offres et requêtes' dans le cadre de l'AGCS"1. Son objectif est d'aider les Membres de l'OMC à mieux connaître les questions qui revêtent une importance particulière pour le secteur des services juridiques et à savoir comment les aborder au cours des négociations.

Le secteur juridique et les échanges de services juridiques n'ont cessé de se développer au cours des dernières décennies suite à l'expansion des échanges internationaux et à l'apparition de nouveaux domaines, comme les secteurs des affaires et du droit commercial. Les modes 3 et 4 revêtent une importance croissante pour les cabinets de consultations juridiques, dont les clients internationaux ont besoin d'avis touchant plusieurs juridictions et d'un service intégré coiffant tous les aspects d'une transaction. Face à cette demande, les cabinets de consultations juridiques cherchent souvent à établir leurs propres réseaux internationaux par le biais d'une présence commerciale et à se doter d'un pool de juristes connaissant un grand nombre de pays, y compris les pays d'accueil, et les pratiques commerciales intéressant leurs clients.

Dans ce secteur, les échanges continuent d'être entravés par toutes sortes de mesures découlant du caractère national du droit en vigueur dans chaque pays. Ces mesures concernent avant tout les modes 3 et 4 , car les autres modes de fourniture sont beaucoup plus difficiles à réglementer.

Bien que la distinction soit parfois difficile à opérer, il importe de distinguer, d'une part, les mesures relatives à l'accès aux marchés et au traitement national et, de l'autre, les mesures de réglementation nationales. Les mesures relatives à l'accès aux marchés et au traitement national donnent lieu à l'établissement de listes conformément aux dispositions des articles XVI et XVII de l'AGCS et, en conséquence, à des négociations visant leur suppression. Des propositions de négociations et des demandes de plusieurs Membres de l'OMC tendent à l'élimination de bon nombre de ces restrictions. Comme, par exemple, il arrive souvent que les cabinets de juristes internationaux n'essaient pas de spécialiser leurs juristes dans le droit en vigueur dans le pays d'accueil, mais cherchent à s'associer à des juristes locaux et à en recruter, les restrictions à l'association avec des spécialistes locaux et à leur recrutement sont considérées comme constituant d'importants obstacles à supprimer. De même, les règles en matière de nationalité et de résidence sont souvent considérées comme trop contraignantes et pourraient être remplacées par des mesures moins restrictives, comme l'assurance responabilité civile.

D'un autre côté, les mesures réglementaires nationales non discriminatoires, comme les prescriptions en matière de qualifications, relèvent de l'article VI de l'AGCS et n'ont pas besoin d'être inscrites dans la Liste. Les services juridiques, à l'instar de bien d'autres services professionnels, ont tendance à être fortement réglementés pour répondre à une série d'objectifs d'intérêt général. Si la légitimité de cette réglementation n'est pas en cause, les Membres de l'OMC sont encouragés à appliquer une réglementation efficace qui n'apporte pas de restrictions non nécessaires aux échanges. De même, les Membres pourraient

OCDE, 2002a. 
être encouragés à étudier des solutions qui facilitent les échanges, telles que la mise en place de systèmes limités de licences pour les conseils juridiques étrangers et des accords de reconnaissance mutuelle.

Si le Cycle d'Uruguay n'a débouché que sur une libéralisation modérée, les négociations en cours offrent l'occasion de faire progresser cette libéralisation dans tous les pays. Les avantages qui en résulteront pourront aller bien au-delà de l'expansion du secteur pour s'étendre à une amélioration de la qualité et à une baisse des prix pour tous les consommateurs de services juridiques (qu'il s'agisse de particuliers ou d'entreprises). Comme il s'agit essentiellement d'entreprises, l'offre accrue de ces services et le climat plus sûr qu'elle génère peuvent contribuer à attirer l'investissement étranger, à créer des débouchés commerciaux pour les fournisseurs locaux et à accroître l'efficience économique d'une manière générale.

Il faut compléter la libéralisation par la mise en place d'un cadre réglementaire approprié pour faire en sorte de préserver les objectifs d'intérêt général, comme la protection des consommateurs. Une libéralisation progressive et transparente permet aussi de se préparer à l'intensification de la concurrence. L'AGCS est bien placé pour poursuivre ces objectifs en donnant aux Membres toute liberté de choisir les modalités de la libéralisation.

Il convient de garder à l'esprit deux questions importantes, qui sont propres au secteur juridique. Premièrement, l'impact de la libéralisation sur le consommateur sera relativement moins grand dans ce secteur car la plupart des échanges de services juridiques se font d'entreprise à entreprise et non d'entreprise au consommateur. En outre, la libéralisation présente peu de risques spéciaux du point de vue de la protection des consommateurs, attendu que les juristes étrangers travaillent dans leur domaine de compétence - c'est-à-dire, lorsqu'ils fournissent des services juridiques liés à leur pays d'origine ou à leurs qualifications internationales, ou lorsqu'un juriste localement qualifié fournit tous les services juridiques locaux pour le compte d'une entreprise étrangère.

Deuxièmement, la libéralisation aura aussi, vraisemblablement, une incidence plus légère sur les fournisseurs locaux, attendu que ceux-ci conservent un avantage compétitif au regard de la pratique du droit national. Qui plus est, les entreprises étrangères sont tenues d'employer des juristes ayant des qualifications locales dès lors qu'elles souhaitent développer leur activité dans le domaine du droit du pays d'accueil.

$\mathrm{Si}$, pour la plupart, les pays en développement n'ont pas actuellement de grands intérêts dans le domaine des exportations de services juridiques, ils gagneraient néanmoins beaucoup à une libéralisation des importations de ces services. En plus d'avantages économiques supplémentaires, tels qu'une plus grande capacité à attirer les investissements étrangers, la présence de fournisseurs étrangers, y compris par la voie d'une coopération entre les entreprises nationales et étrangères et l'emploi de juristes locaux par les entreprises étrangères, peut contribuer à créer un secteur plus compétitif de par le fait qu'ils mettent les juristes locaux en mesure d'acquérir des connaissances et une expertise. Cela peut ensuite contribuer à la formation d'une capacité d'exportation. L'AGCS et, en particulier les articles IV et XIX, confère aux pays en développement une flexibilité supplémentaire pour poursuivre ces objectifs. Il peut aussi être particulièrement intéressant pour ces pays de leur accorder, à l'appui de la libéralisation, une assistance technique et un renforcement de leurs capacités. 


\section{INTRODUCTION}

1. Comme convenu par le Groupe de travail du Comité des échanges à sa réunion du 14 octobre 2002, le présent document fait partie des travaux entrepris par l'OCDE en coopération avec la CNUCED en vue d'aider les Membres de l'OMC à mener avec succès des négociations sur les offres et requêtes dans le cadre de l'AGCS ${ }^{2}$. Il cherche en particulier à donner une plus grande spécificité aux listes de négociation génériques établies dans la partie II du document "Organisation des négociations fondées sur le principe des 'offres et requêtes' dans le cadre de l'AGCS"'3 , en les appliquant aux services juridiques. Il a pour objectif fondamental d'aider les Membres de l'OMC à mieux déterminer les questions revêtant une importance particulière dans le secteur des services juridiques ainsi que la manière dont on pourrait les aborder au cours des négociations.

2. La nécessité de relever le degré de libéralisation consolidée dans le cadre de l'AGCS est considérée comme une des principales tâches auxquelles les Membres de l'OMC sont confrontés dans le domaine des services juridiques. La raison en est à la fois que les négociations d'Uruguay n'ont procuré qu'une faible moisson d'engagements en matière de libéralisation et que, au mieux, les Membres ont maintenu les restrictions déjà en vigueur. Les négociations actuelles sur l'AGCS offrent aux Membres de l'OMC à tous les niveaux de développement la possibilité de réaliser des avancées en matière de libéralisation de manière souple et progressive.

3. On mesure de façon croissante les avantages que peut procurer une plus grande ouverture du marché dans le domaine des services, notamment dans celui des services juridiques. Simultanément, on s'aperçoit de plus en plus que l'ouverture du marché des services à la concurrence étrangère n'est pas tâche aisée. Elle suppose toute une panoplie de mesures, d'instruments de réglementation, d'institutions et de groupes, nationaux comme étrangers. Elle oblige à veiller tout particulièrement à déterminer la nature, le rythme et l'ordre chronologique des engagements en matière de libéralisation et des réformes réglementaires afin de pouvoir à la fois tirer parti de l'ouverture accrue du marché et s'assurer que les objectifs d'intérêt général, comme la protection des consommateurs, sont atteints. Ces problèmes peuvent présenter une acuité particulière pour les pays en développement qui, en moyenne, risquent davantage d'être démunis en matière de régime de réglementation et de capacité de mise en œuvre et qui, partant, demandent à recevoir un niveau suffisant d'assistance technique et d'aide au renforcement de leurs capacités.

4. La liste de référence sur les services juridiques proposée dans le présent document a pour objet principal de faciliter la tâche des hauts responsables des Membres de l'OMC en mettant en relief certaines des grandes questions qu'ils pourront vouloir examiner en formulant et en évaluant les offres et les requêtes. Cette liste n'a toutefois qu'un caractère indicatif. Étant donné la grande diversité des intérêts économiques, du potentiel d'exportation et des besoins de développement des Membres de l'OMC, il faut affiner cette liste pays par pays afin d'en rehausser l'intérêt pratique.

2 Dans le cadre de ce projet conjoint OCDE-CNUCED, plusieurs listes sectorielles ont été établies sur les services d'assurance (OCDE, 2003a) et du secteur de l'énergie (CNUCED, 2003). Les secrétariats de l'OCDE et de la CNUCED vont achever en 2004 deux autres listes concernant respectivement les services environnementaux et les services de construction. 
5. Après une vue d'ensemble du secteur des services juridiques présentée dans la section suivante, la section III expose certaines des tendances qui y sont observées, ainsi que les principales mesures qui restreignent actuellement les échanges de ces services. La section IV examine les faits nouveaux touchant l'AGCS, et notamment les questions relatives aux définitions, les engagements en vigueur et l'évolution des négociations en cours. La section $\mathrm{V}$ analyse ensuite les avantages potentiels d'une plus grande ouverture du marché des services juridiques, les problèmes qu'il convient d'aborder dans les négociations actuelles sur l'accès à ce marché, et les principales questions qui se posent dans le domaine de la réglementation intérieure. Enfin, une annexe présente une liste de questions que les Membres de l'OMC voudront peut-être examiner dans le cadre de la procédure des négociations fondées sur le principe des offres et requêtes.

\section{CROISSANCE DU SECTEUR DES SERVICES JURIDIQUES}

6. Les services juridiques jouent un rôle crucial dans la facilitation des affaires et sont un élément vital de l'infrastructure qui sous-tend le commerce mondial. On ne peut envisager de procéder à des opérations commerciales, en particulier de caractère trans-national, sans l'intervention de juristes. Les cabinets de consultations juridiques de nombreux pays s'internationalisent à mesure que leurs clients cherchent des débouchés sur un marché qui se mondialise rapidement et ont besoin d'un niveau continuel de services recouvrant diverses juridictions ${ }^{4}$. Si ces services ne peuvent être offerts par le pays lui-même, la plus grande partie du travail juridique que suppose toute opération transnationale se fera à l'étranger.

7. Au cours des dernières décennies, le secteur des services juridiques n'a cessé de se développer en raison de l'expansion des échanges et de l'apparition de nouveaux domaines du droit relevant, notamment, du droit des affaires et du droit commercial. Des secteurs, comme la restructuration des entreprises, la privatisation, les fusions et acquisitions transnationales, les droits de propriété intellectuelle et le droit de la concurrence, ont créé une demande croissante de services juridiques plus pointus. Il en est aussi résulté l'apparition d'une nouvelle catégorie de juristes chargés principalement de fournir des services consultatifs - par opposition aux avocats traditionnels qu'on voit dans les tribunaux nationaux - , qui sont censés conseiller leurs clients sur des opérations et investissements intervenant partout dans le monde ${ }^{5}$, et assumer des rôles joués traditionnellement par les banques d'investissement (en particulier pour la coordination de transactions globales).

8. Malgré la rareté de données fiables sur l'ampleur générale du secteur, l'OMC estime que dans la Communauté européenne le nombre de prestataires professionnels de services juridiques a progressé en moyenne de plus de $20 \%$ au cours de la période 1989-1993, tandis qu'aux États-Unis il a triplé entre 1973 et $1993^{6}$. En 1999, le nombre de juristes a atteint 858000 aux États-Unis et la pratique du droit est passée d'un montant estimatif de 4.2 milliards de dollars par an en 1965 au chiffre estimatif de 148 milliards par

\footnotetext{
OMC, 2001a.

OCDE, 1996; et OMC, 1998a.

OMC, 1998a.
} 
an en $1999^{7}$. En 2000, l'on comptait 617060 juristes dans la Communauté européenne et, en 1999, les services juridiques fournis ont atteint 176 milliards d'euros ${ }^{8}$.

9. Le secteur des services juridiques a aussi été marqué par une importante consolidation au cours de la dernière décennie, avec pour conséquence la création d'un nombre croissant de cabinets de consultations juridiques multinationaux. Entre 1997 et 2002, les effectifs des 50 cabinets les plus importants ont progressé de $51 \%$, contre $62 \%$ pour leurs recettes (voir le graphique 1 ci-après). Le Global 100, établi chaque année par Legal Business et l'American Lawyer, indique qu'en 2002, classés d'après leurs recettes, les 98 premiers cabinets de consultations juridiques se trouvaient aux États-Unis (69), au Royaume-Uni (17), en Australie (7) et au Canada (5). La liste ne contenait que deux cabinets non anglosaxons, tous deux situés en Espagne ${ }^{9}$. Les grands cabinets internationaux restent donc, pour la plupart, limités à quelques pays de droit commun anglo-saxons. Cela dit, les juristes de ces cabinets ont toutes les chances d'être des ressortissants de nombreux pays différents, y compris de pays en développement, ou d'être spécialisés dans le droit de ces pays. Les grands cabinets de consultations juridiques internationaux recrutent dans le monde entier: les effectifs de Baker \& McKenzie, $\mathrm{N}^{\circ} 2$ du Global 100 de 2002, proviennent de plus de 60 pays. ${ }^{10}$ Par ailleurs, au cours des dernières années, un nombre accru de grands cabinets ont aussi fait leur apparition dans des juridictions de droit écrit, comme la France, l'Allemagne et les Pays-Bas ${ }^{11}$.

10. Un nouvel aspect du marché des services juridiques internationaux est l'expansion des cabinets de consultations juridiques asiatiques et l'apparition, dans ces cabinets, d'une activité à l'exportation. À Hong Kong (Chine), par exemple, les recettes totales du secteur juridique dépassent 1.5 milliards USD. Hong Kong compte actuellement 569 cabinets de consultations juridiques, dont 49 étrangers, qui emploient 4179 conseillers juridiques, et 114 qui emploient 732 avocats du barreau ${ }^{12}$. En 2000, on a estimé à 849 millions de dollars de Singapour les recettes totales des cabinets de consultations juridiques nationaux de ce pays ${ }^{13}$.

$7 \quad$ Association du barreau de l'État de New York, 2000.

8 Eurostat, 2002.

9 International Financial Services, Londres, 2003. Le tableau 2 de l'annexe donne le classement des 50 plus grands cabinets de consultations juridiques mondiaux.

$10 \quad$ Baker \& McKenzie, 2003.

11 OMC, 1998a.

12 Lowtax.net, 2003. Un avocat du barreau est un spécialiste du contentieux dont l'activité se limite à plaider devant les tribunaux. Un conseil juridique se limite à donner des conseils juridiques et, en règle générale, ne plaide pas lui-même.

13 Ministère des Affaires juridiques, Singapour, 2003. 


\section{Graphique 1. Croissance des services juridiques}

Pourcentage de croissance des 50 plus grands cabinets, 1997/98-2001/02

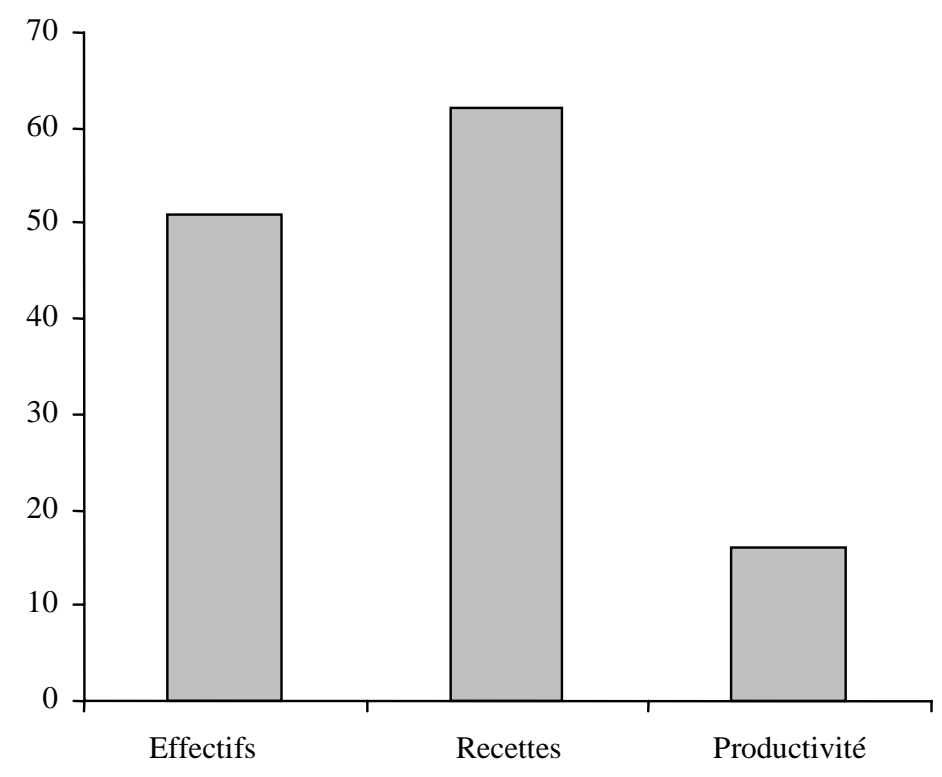

Source : The American Lawyer et Legal Business

\section{COURANTS D'ECHANGES DE SERVICES JURIDIQUES ET MESURES RESTREIGNANT CES ECHANGES}

11. Ces dernières années, peut-être comme suite à l'expansion du commerce international et à l'apparition de nouveaux domaines d'activité, les échanges de services juridiques semblent avoir progressé à un rythme encore plus soutenu que l'ensemble du secteur. Les deux principaux exportateurs sont les États-Unis et le Royaume-Uni. En 1999, les États-Unis ont exporté des services juridiques pour une valeur de 2.56 milliards USD, contre 97 millions USD en 1986, la valeur de leurs exportations étant ainsi multipliée par plus de 26 en 13 ans $^{14}$. En 2002, le Royaume-Uni a exporté pour 1.838 milliards GBP de services juridiques, ce qui équivaut approximativement au montant des exportations britanniques de services de communication et représente un quasi doublement des exportations de services juridiques depuis $1997^{15}$. Les échanges de services juridiques ont aussi progressé sensiblement dans d'autres pays. À Hong Kong, les exportations chinoises de services juridiques, par exemple, se sont chiffrées en 2001 à 817

14 Terry, 2001.

15 Royaume-Uni, Office of National Statistics, 2003. Il convient de noter que ces chiffres ne prennent pas en compte les recettes des 600 filiales de cabinets de consultations juridiques britanniques établies dans différents pays du monde. 
millions HKD (105 millions USD), en hausse de $87 \%$ par rapport à $2000^{16}$. De même, les exportations australiennes de services juridiques sont passées de 74 millions AUD en 1987/88 à quelque 250 millions AUD en $2000 / 01^{17}$.

12. Le droit des affaires et le droit international sont les branches du droit qui donnent lieu au plus d'échanges internationaux de services juridiques, l'essentiel de la demande de services dans ces deux domaines du droit émanant des entreprises et des organismes qui procèdent à des transactions internationales. Les hommes de loi étrangers, pour la plupart, fournissent des services juridiques consultatifs sur le droit de leur pays d'origine, le droit de tout pays tiers dans lequel ils sont compétents, ou le droit international. Jusqu'ici, le droit interne (celui du pays d'accueil) n'a joué qu'un rôle marginal en raison des prescriptions relatives aux qualifications qui, comme le droit interne, s'inscrivent dans un schéma national ${ }^{18}$. Dans ce secteur des échanges de services, le droit du pays d'accueil gagne toutefois du terrain, les cabinets de consultations juridiques ou les juristes étrangers s'efforçant d'être à même de s'associer avec des juristes locaux ou de faire appel à leurs services, plutôt que de chercher à acquérir euxmêmes les qualifications exigées localement..

13. Le commerce des services juridiques peut franchir les frontières (mode de fourniture 1) et profiter dans une très large mesure de l'expansion de l'Internet et du commerce électronique, bien que les prescriptions en matière de résidence et de présence commerciale risquent de limiter ce potentiel de croissance. La prestation de services peut également avoir lieu pendant le séjour temporaire de personnes physiques se déplaçant à titre professionnel indépendant (mode de fourniture 4). L'établissement d'une présence commerciale sous la forme de la mise en place de cabinets de consultations juridiques dans d'autres juridictions (mode de fourniture 3), bien que limité dans le passé, progresse sensiblement. Les cabinets de consultations juridiques anglais, par exemple, ont actuellement quelque 600 filiales à l'étranger et une cinquantaine de pays ont une représentation juridique à Londres. En Chine, 189 cabinets de consultations juridiques étrangers ont établi une présence commerciale.

14. La présence commerciale et la présence de personnes physiques intéressent tout particulièrement les services juridiques modernes, notamment en raison de l'augmentation considérable de la demande de conseils multijuridictionnels (qui supposent souvent une connaissance de l'environnement local) et de services entièrement intégrés portant sur la totalité des aspects d'une transaction. Les cabinets de consultations juridiques qui cherchent à satisfaire les aspirations commerciales de sociétés internationales tentent souvent de constituer leurs propres réseaux internationaux grâce à leur présence commerciale, afin d'avoir une couverture géographique suffisante pour pouvoir répondre aux besoins de leurs clients. Ils s'efforcent aussi de constituer un réseau de juristes qui connaissent les nombreux pays (dont le pays d'accueil), pratiques et conventions internationales intervenant dans les affaires de leurs clients ${ }^{19}$.

15. Les échanges de services juridiques sont touchés par toute une série de mesures, dont beaucoup tiennent à ce que la profession juridique est fragmentée par les frontières nationales et reflète le caractère national du droit de chaque pays. La plupart des Membres de l'OMC imposent de sévères restrictions à la présence commerciale et à la présence de personnes physiques. Les autres modes de fourniture transfrontières et consommation à l'étranger - sont soumis à des restrictions moins rigoureuses parce qu'ils sont beaucoup plus difficiles à réglementer. En plus des restrictions imposées à l'accès au marché et au traitement national, les mesures de réglementation intérieure telles que les critères de qualification

\footnotetext{
16 Tdctrade.com, 2003.

$17 \quad$ Miller et Gallacher, 2002.

18 OMC, 1998a; et Miller and Gallacher, 2002.

19 Department for Constitutional Affairs, Royaume-Uni, 2002.
} 
peuvent aussi influer fortement sur les échanges de services juridiques. Le tableau 1 présente certaines des principales mesures qui influent sur le commerce des services juridiques suivant les modes de fourniture 3 et 4 : celles que les fournisseurs peuvent rencontrer dans d'autres secteurs (mesures horizontales) comme celles qui sont propres à ce secteur.

Tableau 1. Mesures affectant le commerce des services juridiques1

\begin{tabular}{|c|c|c|c|}
\hline $\begin{array}{l}\text { Types de } \\
\text { mesures }\end{array}$ & Mode de fourniture 3 & Mode de fourniture 4 & Modes de fourniture 3 et 4 \\
\hline $\begin{array}{l}\text { Accès aux } \\
\text { marchés } \\
\text { (art. XVI) }\end{array}$ & $\begin{array}{l}\text { Restrictions concernant la } \\
\text { forme juridique. Elles } \\
\text { interdisent ou restreignent la } \\
\text { pratique du droit par les } \\
\text { personnes morales afin que } \\
\text { les spécialistes ne limitent ni } \\
\text { ne contournent leurs } \\
\text { responsabilités } \\
\text { professionnelles. } \\
\text { Restrictions au partenariat } \\
\text { avec les professionnels } \\
\text { titulaires d'un diplôme local } \\
\text { ou au recrutement de } \\
\text { professionnels locaux. } \\
\text { Eviter que des cabinets de } \\
\text { consultations juridiques } \\
\text { étrangers puissent } \\
\text { représenter leurs clients } \\
\text { devant les tribunaux et } \\
\text { exercer leur activité dans le } \\
\text { domaine du droit du pays } \\
\text { d'accueil en s'associant avec } \\
\text { des juristes qualifiés locaux } \\
\text { ou en en employant.2 }\end{array}$ & $\begin{array}{l}\text { Restrictions concernant le } \\
\text { mouvement de personnel, } \\
\text { telles qu'examen des } \\
\text { besoins économiques et du } \\
\text { marché du travail. Ces } \\
\text { restrictions peuvent s'appliquer } \\
\text { aux personnes cherchant à } \\
\text { s'établir pour une durée } \\
\text { relativement longue grâce à } \\
\text { une forme de présence } \\
\text { commerciale relevant du } \\
\text { mode } 3 \text { (cessionnaires entre } \\
\text { sociétés d'un même nom) ou } \\
\text { aux personnes physiques } \\
\text { effectuant un voyage d'affaires } \\
\text { de courte durée (fournisseurs } \\
\text { de services contractuels). }\end{array}$ & $\begin{array}{l}\text { Prescriptions concernant la } \\
\text { nationalité } \\
\text { Ces prescriptions sont plus } \\
\text { fréquentes dans la pratique du droit } \\
\text { interne (conseils et plaidoiries). En } \\
\text { droit international et dans le droit des } \\
\text { pays d'origine et des pays tiers, les } \\
\text { services consultatifs sont rarement } \\
\text { visés par ces dispositions, bien qu'ils } \\
\text { puissent ne pas être ouverts aux } \\
\text { étrangers en présence d'une } \\
\text { prescription globale concernant les } \\
\text { services juridiques. }\end{array}$ \\
\hline $\begin{array}{l}\text { Traitement } \\
\text { national } \\
\text { (Art. XVII) }\end{array}$ & & & $\begin{array}{l}\text { Prescriptions concernant la } \\
\text { résidence. Elles peuvent revêtir la } \\
\text { forme de résidence préalable, de } \\
\text { résidence permanente et de } \\
\text { domicile.3 La condition de la } \\
\text { résidence préalable confère un } \\
\text { avantage compétitif aux fournisseurs } \\
\text { de services qui résident déjà dans le } \\
\text { pays d'accueil depuis plusieurs } \\
\text { années et qui, dans leur grande } \\
\text { majorité, en sont ressortissants. La } \\
\text { résidence permanente, bien que } \\
\text { pouvant être moins restrictive, } \\
\text { impose aussi une charge } \\
\text { additionnelle aux fournisseurs de } \\
\text { services étrangers qui, contrairement } \\
\text { aux fournisseurs nationaux qui y } \\
\text { résident déjà, doivent obtenir le droit } \\
\text { de résidence dans le pays d'accueil. } \\
\text { Dans le cas de personnes physiques } \\
\text { cette disposition peut aussi } \\
\text { leur faire perdre le droit de résidence } \\
\text { dans leur pays d'origine. L'obligation } \\
\text { d'être domicilié dans le pays }\end{array}$ \\
\hline
\end{tabular}




\begin{tabular}{|c|c|c|c|}
\hline & & & $\begin{array}{l}\text { d'accueil, c'est-à-dire d'y avoir une } \\
\text { adresse, n'a que des effets de } \\
\text { distorsion minimes sur les échanges } \\
\text { (du moins pour les modes } 3 \text { et } 4 \text {.) }\end{array}$ \\
\hline $\begin{array}{l}\text { Réglemen- } \\
\text { tation } \\
\text { intérieure } \\
\text { (Art. VI :4) }\end{array}$ & $\begin{array}{l}\text { Restrictions concernant } \\
\text { I'utilisation des raisons } \\
\text { sociales de sociétés } \\
\text { internationales et } \\
\text { étrangères. Elles sont } \\
\text { énumérées dans les } \\
\text { prescriptions en matière de } \\
\text { licences qui figurent dans les } \\
\text { Disciplines de l'OMC relatives } \\
\text { au secteur des services } \\
\text { comptables. Elles peuvent } \\
\text { toutefois constituer une limite } \\
\text { à l'application du traitement } \\
\text { national si elles modifient les } \\
\text { conditions de concurrence } \\
\text { entre les fournisseurs de } \\
\text { services locaux et étrangers } \\
\text { au sens de l'article XVII de } \\
\text { l'AGCS. }\end{array}$ & & $\begin{array}{l}\text { Prescriptions concernant les } \\
\text { qualifications4. Ces prescriptions } \\
\text { sont particulièrement rigoureuses } \\
\text { dans la pratique du droit du pays } \\
\text { d'accueil, mais elles peuvent aussi } \\
\text { être appliquées au droit du pays } \\
\text { d'origine et de pays tiers, ainsi qu'au } \\
\text { droit international. Elles servent à } \\
\text { faire en sorte que les professionnels } \\
\text { étrangers soient qualifiés et diplômés } \\
\text { dans le domaine du droit où ils se } \\
\text { proposent d'exercer leur activité } \\
\text { dans le pays d'accueil. }\end{array}$ \\
\hline Autres & & $\begin{array}{l}\text { Procédures administratives } \\
\text { relatives à la délivrance de } \\
\text { visas et de permis de travail. } \\
\text { Elles font partie de la politique } \\
\text { d'un pays en matière } \\
\text { d'immigration ou d'emploi et } \\
\text { bénéficient d'un traitement à } \\
\text { part conformément à l'Annexe } \\
\text { de l'AGCS sur le mouvement } \\
\text { des personnes physiques. } 5\end{array}$ & \\
\hline
\end{tabular}

1. Plusieurs des mesures mentionnées dans ce tableau, comme les prescriptions concernant la nationalité ou la résidence, influent aussi sur les modes 1 et 2.

2. Ces mesures peuvent aussi être considérées comme concernant le traitement national, car elles peuvent modifier les conditions de la concurrence en faveur des cabinets de consultations juridiques du pays d'accueil.

3. Dans tous ces cas, où les prescriptions concernant la résidence constituent un traitement formellement identique des fournisseurs de services étrangers et des fournisseurs de services nationaux, il faudra déterminer chaque fois si ces prescriptions affectent les conditions de la concurrence au sens de l'article XVII. A cette fin, des facteurs tels que la nature des services juridiques fournis (représentation, conseils, activités notariales, etc.), le mode de fourniture et le type de résidence exigé devront être pris en compte (voir OMC, 1998a).

4. Lorsque les prescriptions imposent une charge supplémentaire aux juristes étrangers, ces mesures devraient être considérées comme des mesures concernant le traitement national.

5. A condition qu'elles ne soivent pas appliquées de manière à annuler ou compromettre des avantages en vertu d'engagements spécifiques. Voir l'Annexe de l'AGCS sur le mouvement des personnes physiques.

Source: OMC, 1998a; et OCDE, 1996. 
16. Au cours des dernières années, des travaux préliminaires ont été entrepris pour quantifier l'effet de différentes restrictions sur une gamme de services professionnels, y compris les services juridiques (voir encadré 1).

\section{Encadré 1. Quantification du coût des restrictions}

Nguyen-Hong (2000) a quantifié les restrictions affectant le commerce des services dans le domaine juridique, comptable, architectural, et celui de l'ingénierie, de 34 économies de par le monde (29 économies pour les services juridiques). Dans son étude, l'auteur a mesuré séparément les restrictions que connaissent une entreprise nationale et une entreprise étrangère au moment d'établir une présence et de poursuivre leurs opérations. Parmi les restrictions mesurées relatives aux services professionnels, les professions avec la plus haute note (c'est-à-dire celles dont les marchés sont les plus restrictifs) sont les services juridiques et comptables, pour les entreprises tant intérieures qu'étrangères, mais surtout pour ces dernières. En réduisant le commerce et la concurrence sur le marché, ces restrictions auront tendance à influer sur le coût et le prix de la prestation des services.

En utilisant des techniques économétriques, l'étude a aussi mesuré l'effet des restrictions aux services d'ingénierie sur les marges entre prix et les coûts. Les résultats portent à croire que les restrictions à la prestation étrangère de services d'ingénierie ont tendance à permettre aux entreprises du marché intérieur de relever leurs prix au-dessus du niveau des coûts. L'incidence des restrictions aux services d'ingénierie a eu pour effet de relever les prix parfois jusqu'à $15 \%$, la plus grande partie de cet accroissement étant imputable aux restrictions à l'établissement d'entreprises étrangères. L'étude attribue aussi une hausse des coûts allant de $1 \%$ à $7 \%$ aux restrictions appliquées aux entreprises nationales. L'indice moyen de restrictivité dans l'échantillon analysé pour les services juridiques est similaire à celui de l'économie la plus restrictive pour les services d'ingénierie. II est donc permis de supputer que l'on constatera un effet similaire sur les prix et les coûts des services juridiques. 


\section{SITUATION ACTUELLE DE L'AGCS}

\section{Définition des services juridiques}

17. La classification sectorielle des services de $\mathrm{l}^{\prime} \mathrm{OMC}^{20}$ ne comporte qu'une entrée pour les services juridiques, qui constituent une subdivision des services professionnels, elle-même une subdivision des services aux entreprises, avec un renvoi à la position 861 de la Classification centrale des produits des Nations Unies (CPC) ${ }^{21}$. Comme l'a souligné le Secrétariat de l'OMC, la distinction faite par la CPC entre les conseils et la représentation en droit pénal, dans d'autres domaines du droit et en procédure réglementaire convenait moins aux Membres souscrivant des engagements que celle qui est faite entre les conseils et la représentation en droit du pays d'accueil, en droit du pays d'origine ou tiers et en droit international $^{22}$.

18. Ces distinctions elles-mêmes sont toutefois en cours de révision dans le cadre des négociations actuelles. Certains Membres de l'OMC ont soumis des propositions contenant des définitions de rechange pour les services juridiques, qui pourraient être utilisées lorsque des pays soumettent leurs offres et leurs requêtes. Ces propositions visent à affiner, améliorer et assouplir la classification actuelle de façon à traduire plus clairement les réalités commerciales des échanges internationaux de services juridiques. Elles cherchent aussi à donner aux Membres la possibilité d'accroître le nombre de leurs engagements en matière de services juridiques et d'en améliorer la qualité sans compromettre les objectifs d'ordre public tels que la protection des consommateurs intérieurs ou le respect de la primauté du droit ${ }^{23}$.

19. Les niveaux que la classification cherche à prendre en compte sont divers, notamment en ce qui concerne les domaines du droit, la catégorie des professionnels et le type de service fourni. Certaines propositions demandaient l'élaboration de définitions communes applicables aux services de consultation juridique étrangers -- c'est-à-dire ceux des professionnels pratiquant le droit international, le droit du pays d'origine ou d'un pays tiers -- et à la pratique du droit international ${ }^{24}$. Selon une proposition, les services juridiques pourraient être subdivisés en sous-secteurs correspondant aux différentes professions (avocats, juges et autres juristes non classés ailleurs) ${ }^{25}$. Une autre a suggéré de définir les services juridiques de façon à y inclure la fourniture de conseils juridiques ou la représentation juridique consistant à donner des conseils dans le cadre de transactions commerciales, à participer à la gestion des organisations

20 Document MTN.GNS/W/120.

21 La révision de la CPC de l'ONU (CPC Rev.1), approuvée par la Commission de statistique de l'ONU en février 1997,/ laisse à peu près inchangés les services juridiques. Elle comprend toutefois comme souscatégorie des services juridiques les "Services d'arbitrage et de conciliation", qui faisaient précédemment partie des services de consultation en gestion.

OMC, 2003a.

OMC, 2003a; et Miller et Gallacher, 2002.

Voir, par exemple, une des communications de l'Australie sur les services juridiques (OMC, 2001a) et celle du Canada sur les services professionnels (OMC, 2001b). 
d'entreprises, à assurer des médiations, à rendre des arbitrages et à fournir des services semblables de règlement non juridictionnel des différends, à entreprendre des campagnes publiques et à exercer des pressions $^{26}$.

20. Plus récemment, une proposition concernant expressément la classification a suggéré d'ajouter 12 nouvelles subdivisions fondées sur le domaine du droit pratiqué et le type de service fourni ${ }^{27}$. Les 12 sous-catégories sont : droit du pays d'origine (services de conseils); droit du pays d'origine (services de représentation); droit d'un pays tiers (services de conseils); droit d'un pays tiers (services de représentation); droit du pays d'accueil (services de conseils); droit du pays d'accueil (services de représentation); droit international (services de conseils); droit international (services de représentation); services d'arbitrage commercial international ; autres services de règlement des différends; rédaction et certification de documents juridiques; et autres services de conseils ou de consultation.

21. Une autre proposition dans ce domaine considère qu'à des fins de classification, le seul paramètre à prendre en compte devrait être la nature des différents services qui peuvent être fournis par des juristes et non les qualifications requises pour exercer les activités correspondantes. A cet égard, en posant comme principe directeur que les fournisseurs de services juridiques ne peuvent fournir de service juridique que dans les domaines du droit pour lesquels ils sont qualifiés, il est proposé : 1) que l'on conserve la classification actuelle des services juridiques (position 861 de la CPC, plus services d'arbitrage et de conciliation) sans autre subdivision ; 2) que l'on remédie à toute limitation de la fourniture de services juridiques, notamment dans les domaines du droit pour lesquels les juristes sont qualifiés pour exercer, à l'aide des colonnes «accès aux marchés » et «traitement national » de leurs listes d'engagements (voir plus loin); et 3) que l'on tienne compte de la possibilité d'ajouter des engagements supplémentaires pour la fourniture de services juridiques dans les domaines du droit pour lesquels le fournisseur de services n'est pas qualifié. $^{28}$

22. L'Association internationale du barreau (IBA) a aussi adopté en septembre 2003 une résolution préconisant un système de terminologie relatif aux services juridiques pour les besoins des négociations commerciales internationales. Cette résolution ne prend pas position sur la question de la classification, mais recommande un système de terminologie relatif aux services juridiques qui puisse être utilisé pour formuler aussi bien des engagements que des réserves à des engagements dans le cadre de l'AGCS. ${ }^{29}$

23. Toutes ces options devront être examinées attentivement par les Membres de l'OMC. En effet, le système de classification utilisé pour le Cycle de Doha influera sensiblement sur l'évolution du marché international des services juridiques. Il est essentiel que la terminologie employée dans le cadre des négociations soit claire et corresponde à la réalité du commerce moderne des services juridiques.

\section{Engagements actuels et futurs}

24. Conformément à l'AGCS, les Membres de l'OMC doivent respecter certaines règles et disciplines générales, qui s'appliquent à chacun d'entre eux et, dans la plupart des cas, à tous les services, dont les services juridiques. Ces règles portent, notamment, sur le traitement de la nation la plus favorisée (NPF) et la transparence; les Membres ont toutefois reçu la possibilité d'établir, une seule fois au cours du cycle d'Uruguay, une liste d'exemptions NPF (possibilité qui a aussi été offerte aux pays qui ont souscrit

\footnotetext{
26 Communication des États-Unis sur les services juridiques (OMC, 2000b).

27 Voir la dernière communication de l'Australie sur les services juridiques (OMC, 2002a).

28 Communication des CE sur les services juridiques (OMC, 2003a).

29

IBA, 2003a.
} 
ultérieurement à l'Accord). Six Membres bénéficient d'exemptions NPF pour les services juridiques ${ }^{30}$ et quatre autres pour les services professionnels ${ }^{31}$. Les raisons avancées pour ces exemptions comprennent l'absence de réciprocité, la protection d'objectifs d'ordre public, tels que la nécessité d'assurer la qualité des services et celle d'approuver l'établissement de cabinets de consultations juridiques étrangers au cas par cas. $^{32}$

25. D'autres obligations concernant l'accès au marché et le traitement national (respectivement articles XVI et XVII) sont également applicables, mais uniquement dans la mesure où un Membre de l'OMC inclut volontairement les services juridiques dans sa "Liste des engagements spécifiques" et sous réserve de toute mesure consignée dans sa liste limitant l'application de ces obligations. Dans le cadre du Cycle d'Uruguay, 45 Membres (en comptant comme un seul les 12 membres de la CE d'alors) ont souscrit des engagements concernant les services juridiques ${ }^{33}$. Sur les 20 Membres qui ont accédé par la suite à l'OMC, tous - sauf la Mongolie - ont pris des engagements dans ce secteur.

26. Pour les services de conseil, le nombre d'engagements figurant sur les listes est bien plus élevé en droit du pays d'origine et en droit international qu'en droit du pays d'accueil ou d'un pays tiers. Les limitations indiquées concernant l'accès aux marchés sont très fréquentes. Plusieurs Membres imposent des prescriptions ou des limitations relatives à la nationalité ou à la citoyenneté aux catégories d'entités juridiques. Quelques Membres ont aussi indiqué dans leurs listes des restrictions au traitement national, notamment en ce qui concerne les prescriptions concernant la résidence. Des mesures de réglementation intérieure non discriminatoires relatives aux prescriptions en matière de licences et de qualifications figurent sur les listes d'un petit nombre de Membres, bien que cette inscription ne soit pas nécessaire et n'exempte pas les mesures considérées de l'application des disciplines visées dans les autres dispositions de l'AGCS.

27. Le Cycle d'Uruguay n'a été que la première étape d'un long processus de réglementation et de libéralisation multilatérales des échanges. Les Membres de l'OMC sont convenus d'engager « des séries de négociations successives...en vue d'élever progressivement le niveau de libéralisation $» .{ }^{34}$ Les négociations sur les services ont commencé ont commencé le $1^{\text {er }}$ janvier 2000 dans le cadre du "programme incorporé »; à Doha, en novembre 2001, les Membres ont décidé d'engager un nouveau cycle global de négociations et de prendre comme point de départ les travaux accomplis depuis 2000 sur les services.

28. Au cours de la première phase des négociations, plusieurs Membres de l'OMC ont présenté des propositions générales exposant leurs intérêts dans des négociations sur les services juridiques. Trois Membres ont soumis des propositions concernant expressément ces services ${ }^{35}$, et quatre Membres, dont un un pays en développement, ont abordé la question des services juridiques indirectement en présentant des

30 Brunéi Darussalam, Bulgarie, Ex-République yougoslave de Macédoine, Lituanie, République dominicaine et Singapour.

31 Costa Rica, Honduras, Panama et Turquie.

32 Voir tableau 3 de l'annexe.

33 Voir tableau 4 de l'annexe.

34 AGCS, article XIX.

35 Communications de l'Australie (OMC, 2002a; OMC, 2001a; et OMC, 2001c); communication des CE (OMC, 2003a); et communications des États-Unis (OMC, 1998b; et OMC, 2000b). 
propositions sur les services professionnels de manière générale ${ }^{36}$. Quelques Membres ont aussi mentionné les services juridiques dans des propositions de négociation multisectorielles ou modales plus générales ${ }^{37}$.

29. Les propositions expriment un point de vue commun selon lequel il importe de faire une distinction entre les mesures visant à assurer la réalisation des objectifs d'ordre public, tels que la protection efficace des consommateurs, et celles qui entravent inutilement le commerce. Les prescriptions concernant la nationalité et la résidence passent souvent pour être plus restrictives pour les échanges que cela n'est nécessaire pour parvenir aux objectifs visés. Il est aussi fait fréquemment mention de l'importance de faciliter l'établissement d'entreprises étrangères (mode de fourniture 3) et le mouvement de praticiens étrangers (mode de fourniture 4) est aussi fréquemment mentionnée, ainsi que de la reconnaissance des qualifications. Plusieurs Membres ont en outre souligné l'importance de la transparence dans ce secteur. (Le tableau 2 énumère plus en détail les principaux éléments des propositions concernant tant les services professionnels en général que les aspects propres aux services juridiques ${ }^{38}$ ).

30. Les Lignes directrices et procédures pour les négociations adoptées par le Conseil du commerce des services de l'OMC et réaffirmées plus tard au paragraphe 15 de la Déclaration ministérielle de Doha ont retenu la stratégie des requêtes et des offres comme étant la principale méthode devant servir à négocier les engagements spécifiques relatifs à l'accès aux marchés des services. À Doha, il a été convenu que les Membres soumettraient les demandes initiales pour le 30 juin 2002 au plus tard et les offres initiales pour le 31 mars 2003.

31. Dans le cadre de cette deuxième phase des négociations, les Membres ont donc échangé leurs requêtes initiales. Comme celles-ci sont communiquées directement entre les Membres concernés de l'OMC et non par l'intermédiaire du Secrétariat de l'Organisation, il n'existe pas de point central rassemblant toutes les demandes. Il est donc impossible de connaître le nombre exact de requêtes, ni d'avoir une vue d'ensemble de leur contenu. Quoi qu'il en soit, il semblerait que quelques Membres ont adressé aux autres des requêtes concernant expressément les services juridiques. La CE, par exemple, qui a publié un résumé de ses demandes, a adressé des requêtes conçues expressément pour l'accès aux marchés des services juridiques à plusieurs Membres de l'OMC.

32. Alors que l'envoi des requêtes se fait de façon bilatérale entre les parties aux négociations, l'usage veut que les offres soient diffusées à l'échelle multilatérale (en raison de la règle NPF). A ce jour, 42 Membres ont soumis des offres initiales ${ }^{39}$. Parmi celles-ci, 12 ont été mises en circulation générale par les Membres concernés et peuvent être consultées sur le site Web de $1^{\prime} \mathrm{OMC}^{40}$. Treize autres peuvent être

36 Communication du Canada (OMC, 2001b); communication de la Colombie (OMC, 2001d); communication des Communautés européennes et de leurs États membres (OMC, 2000c); et communication de la Suisse (OMC, 2001e).

37 Voir, par exemple, la communication de l'Inde sur le mouvement de professionnels (OMC, 2000a).

Le tableau ne mentionne pas les questions de définition, qui ont été abordées dans la section précédente de la présente note.

Argentine; Australie ; Bahreïn; Bolivie ; Bulgarie ; Canada ; Chili ; Chine ; Colombie ; Communautés européennes et leurs États membres ; Costa Rica ; Etats-Unis ; Fidji ; Guatemala ; Hong Kong, Chine ; Inde ; Islande ; Israël ; Japon ; Liechtenstein ; Macao, Chine; Mexique ; Norvège; Nouvelle-Zélande ; Panama ; Paraguay ; Pérou ; Pologne ; République de Corée ; République slovaque ; République tchèque ; Saint-Kittset-Nevis ; Sénégal ; Singapour ; Slovénie ; Sri Lanka ; Suisse ; Suriname ;Taipei chinois ; Thaïlande ; Turquie et Uruguay.

Australie ; Canada ; Chili ; Communautés européennes et leurs États membres ; Etats-Unis ; Islande ; Japon ; Liechtenstein ; Norvège ; Nouvelle-Zélande ; Slovénie ; Turquie. 
consultées sur des sites Web nationaux ou autres ${ }^{41}$. Il appartient au membre individuel de décider s'il rendra son offre publique ou non. Dans les 25 offres rendues publiques, huit Membres offrent d'inclure des modifications dans leurs engagements actuels concernant les services juridiques ${ }^{42}$. Aucun membre n'a offert de prendre de nouveaux engagements dans ce secteur ${ }^{43}$.

33. Les Membres qui ont offert d'apporter des modifications à leurs listes l'ont fait dans leurs engagements spécifiques relatifs aux services juridiques et, dans certains cas aussi, dans leurs engagements horizontaux. Certains Membres ont supprimé les restrictions à l'accès aux marchés et au traitement national pour ce qui est des prescriptions concernant la nationalité ou la résidence ou ont assoupli les restrictions relatives à la forme juridique. D'autres ont levé les restrictions à la formation de partenariats avec des cabinets de consultations juridiques locaux ou à l'engagement de professionnels locaux. Dans certains cas, la levée des restrictions ne concerne que quelques juridictions dans un pays particulier. Certaines offres comprennent aussi des spécifications relatives à la transparence visant à protéger le consommateur, telles que l'obligation de souscrire une assurance responsabilité ou de respecter les codes déontologiques du pays.

34. D'autres pays ont levé les mesures restreignant le champ d'activité, permettant ainsi à des étrangers de fournir des services juridiques dans les domaines du droit pour lesquels ils sont qualifiés. En pareil cas, les engagements prévoient des spécifications relatives à la transparence (ils stipulent, par exemple, que la pratique du droit du pays d'accueil pourra être subordonnée à une inscription au barreau local) et des limites à l'accès aux marchés pour les étrangers nouvellement admis à exercer le droit du pays d'accueil (concernant, par exemple, les types de forme juridique). Plusieurs pays ont aussi assoupli les dispositions réglementaires intérieures et facilité la reconnaissance des qualifications entre les juridictions (par exemple, l'obligation d'avoir une formation minimale et une expérience de la pratique du droit du pays d'accueil et du droit international) ${ }^{44}$.

$41 \quad$ Argentine; Bulgarie; Colombie; Hong Kong (Chine); Inde; Israel; Mexique ; Panama ; Paraguay ; Pologne ; Singapour ; Suisse et Uruguay.

42 Australie ; Canada ; Communautés européennes et leurs Etats membres ; Islande ; Japon ; Pologne ; Suisse et Turquie.

Toutefois, la Corée proposerait dans son offre - bien que non disponible publiquement - de prendre pour la première fois des engagements concernant l'accès aux marchés des services juridiques. Voir UK Government, 2003.

Comme indiqué précédemment, toutefois, l'inscription des règlements non discriminatoires n'est pas nécessaire. 


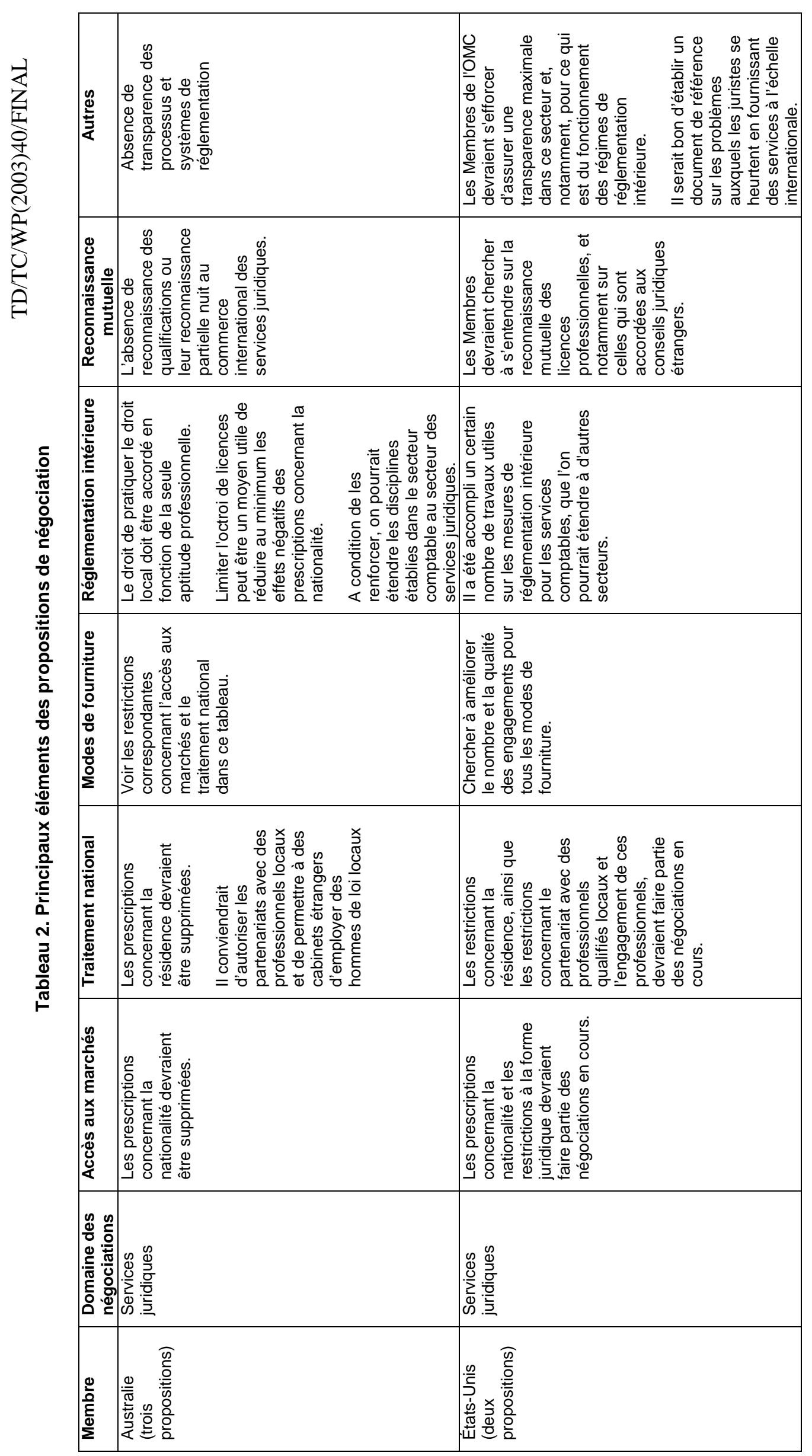




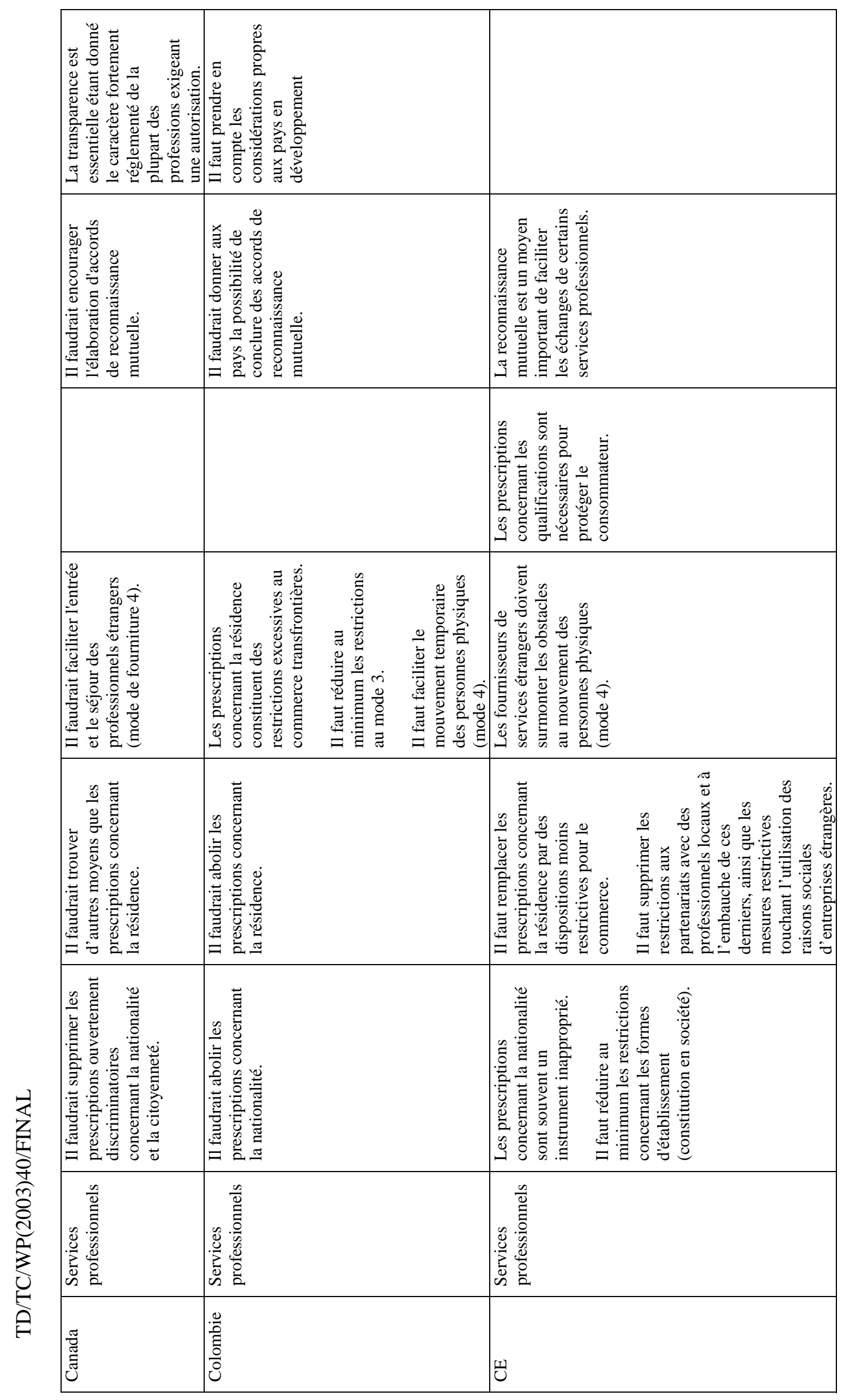




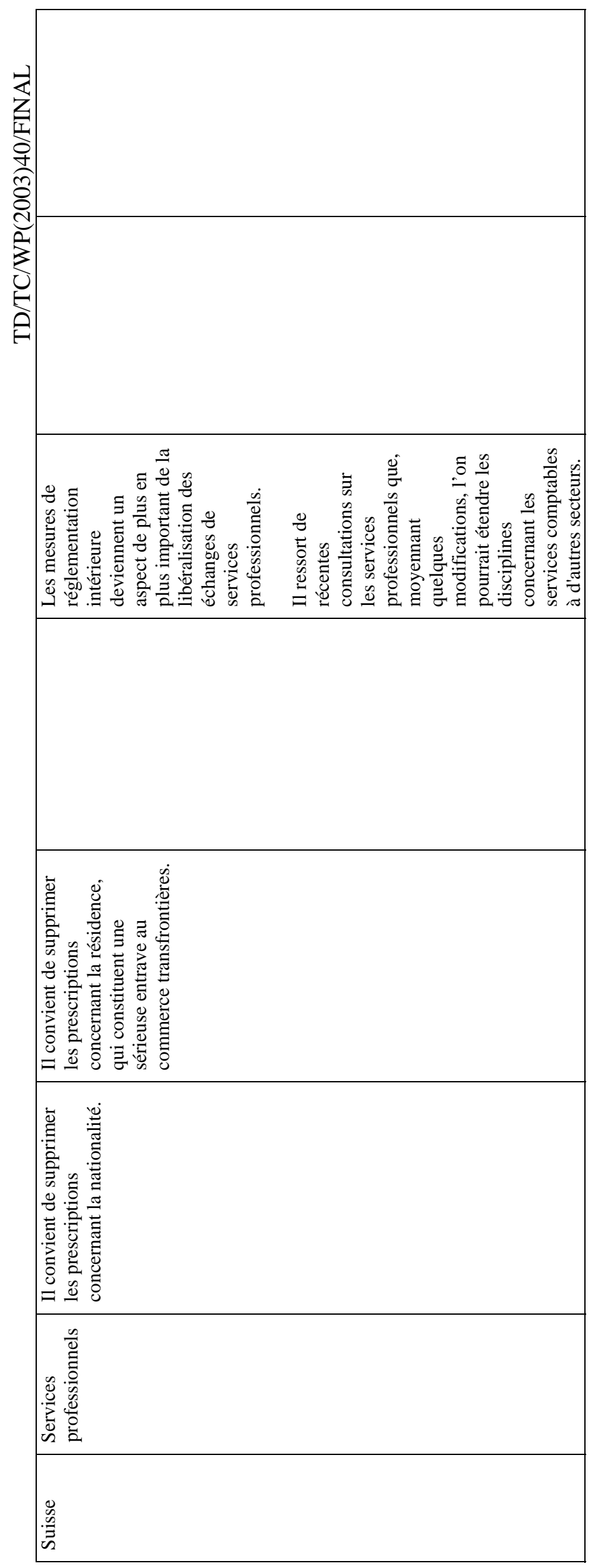




\section{AVANTAGES ESCOMPTES, PROBLÈMES À RESOUDRE ET EFFORTS DE RÉFORME DE LA RÉGLEMENTATION A ENTREPRENDRE}

\section{Avantages potentiels de l'ouverture des marchés pour les services juridiques}

35. Il ne faut pas sous-estimer l'importance de la libéralisation des échanges de services juridiques et des investissements dans ce domaine. Non seulement la libéralisation peut ouvrir de nouveaux débouchés aux fournisseurs de services juridiques, mais elle peut contribuer au développement des échanges dans leur ensemble. De fait, sans l'intervention des hommes de loi, les échanges de services comme ceux que de marchandises ne se dérouleraient pas de manière méthodique, sûre et prévisible. De plus en plus, les hommes de loi jouent un rôle fondamental en facilitant les échanges dans l'économie mondiale et sont considérés comme faisant partie de l'infrastructure globale du commerce ${ }^{45}$.

36. Le commerce des services juridiques, comme celui de beaucoup d'autres services, remet en cause la notion très répandue selon laquelle les exportations sont bénéfiques pour l'économie nationale, contrairement aux importations. En réalité, l'importation de services juridiques peut, directement ou indirectement, présenter d'importants avantages. Le consommateur national peut y trouver son compte grâce à une offre plus vaste de services juridiques, à l'amélioration de leur qualité et à une réduction de leur prix, l'ouverture du marché des services juridiques aux hommes de loi et aux cabinets de consultations juridiques étrangers contribuant à rendre ce marché plus compétitif ${ }^{46}$.

37. Les clients, tant nationaux qu'internationaux, peuvent aussi tirer certains gains d'efficience du principe d'un "guichet unique" dans leur juridiction nationale, guichet doté de compétences dans des ressorts multiples, par opposition à l'inefficience de marchés de services morcelés par systèmes nationaux ${ }^{47}$. Le système commercial lui-même peut s'améliorer grâce à la facilitation des transactions et à l'aptitude des hommes de loi à contrôler les réformes de la réglementation et du marché sur la base de normes internationales ${ }^{48}$.

38. Le commerce international des services juridiques peut aussi être considéré comme un catalyseur de l'investissement étranger, en contribuant à la fiabilité et à la prévisibilité du climat commercial local. Face à l'augmentation rapide des investissements transfrontaliers et à la quête de nouvelles sources de capital, les investisseurs étrangers ont besoin de stabilité et de prévisibilité pour savoir à quels risques ils s'exposent et rentabiliser leurs investissements. Dans le cadre de ce processus, ils ont souvent recours aux services de juristes expérimentés, pouvant leur garantir des services de qualité quel que soit le pays concerné $^{49}$. L'existence de juristes pouvant offrir une panoplie plus complète et plus élaborée de services

\footnotetext{
45 Department for Constitutional Affairs, Royaume-Uni, 2003a.

46 Johnston, 2002.

47 Johnston, 2002.

48 Department for Constitutional Affairs, Royaume-Uni., 2003b.

49 Department for Constitutional Affairs, Royaume-Uni., 2003a.
} 
peut aussi attirer un nombre croissant de clients internationaux ${ }^{50}$ et déboucher sur la pratique locale de nouvelles formes de droit tels que l'arbitrage et la conciliation.

39. Grâce à différentes formes de collaboration entre les bureaux de consultations juridiques étrangers et nationaux, ainsi qu'à l'engagement de juristes locaux par des cabinets étrangers, la présence d'hommes de loi étrangers peut créer des emplois pour les praticiens locaux. Elle peut aussi créer un secteur plus compétitif et plus dynamique permettant aux cabinets et avocats nationaux d'assimiler des innovations, des connaissances et des compétences qu'ils peuvent partager avec leurs homologues étrangers. À mesure que les cabinets de consultations juridiques nationaux se renforceront et deviendront plus concurrentiels, ils pourront aussi devenir compétents dans le domaine des exportations. L'efficacité globale accrue résultant de la libéralisation peut en fait aider les prestataires de services nationaux à se positionner de façon à tirer parti de l'expansion du marché dans le secteur des services juridiques.

40. Un pays peut aussi tirer des avantages non économiques de son ouverture aux influences extérieures. Dans certains pays où les procédures judiciaires sont très lentes, on peut avoir intérêt à apprendre ce que les autres font pour améliorer l'administration de la justice. On peut aussi avoir avantage à adopter les pratiques suivies dans d'autres juridictions en matière de qualification et de formation permanente des hommes de loi, ou à examiner la façon dont ils défendent les intérêts de leurs clients ${ }^{51}$.

\section{Questions à examiner au cours des négociations}

\section{i) Problèmes relatifs à la libéralisation}

41. Comme indiqué à la section III, malgré son importance croissante, le commerce des services juridiques continue d'être entravé par différentes mesures relatives à l'accès aux marchés et au traitement national, qui interdisent ou réduisent sensiblement la possibilité de faire appel à des fournisseurs étrangers de ces services. Même si leurs objectifs sont souvent similaires, il faut distinguer ces restrictions des mesures de réglementation intérieure qui sont abordées plus loin à la section V. $3^{52}$.

42. La première considération sur laquelle s'appuient bien des restrictions a trait au « service public » qu'accomplissent les praticiens du pays d'accueil qui exercent dans le domaine du droit de leur pays. Il s'agit au fond de règles visant à faire en sorte que les services juridiques soient fournis avec les connaissances et les compétences nécessaires que peuvent en attendre les clients et, notamment, avec la connaissance de la langue, de la culture, des coutumes, des règles et de la situation locales (prescriptions concernant la nationalité ou la résidence, par exemple). D'autres règles s'appuient sur le refus des responsables de la réglementation de reconnaître aux hommes de loi étrangers la qualité d' "hommes de loi » et relèvent de l'interdiction générale d'exercer dans le domaine du droit en partenariat avec qui que ce soit qui n'est pas juriste qualifié, interdiction qui vise à garantir la qualité des services ou l'indépendance des professionnels (restrictions aux partenariats avec des professionnels diplômés locaux ou à l'engagement de personnels de cette catégorie). Une autre grande considération relative à la réglementation concerne la responsabilité du fournisseur de services professionnels à l'égard de ses clients (prescriptions concernant la forme juridique et la résidence, par exemple). Il peut également être important de garantir aux clients la proximité et l'offre de services juridiques (prescriptions concernant la résidence, par exemple).

43. L'offre de services juridiques fait ressortir à l'évidence la légitimité de protéger l'intérêt public. Les efforts de réforme doivent prendre en compte le rôle légitime des gouvernements d'intervenir pour

\footnotetext{
50 Department for Constitutional Affairs, Royaume-Uni., $2003 \mathrm{~b}$.

51 Hook, 2003.

52 Les mesures de réglementation intérieure peuvent être considérées comme des mesures relatives à l'accès aux marchés et au traitement national si elles tombent sous le coup des articles XVI et XVII.
} 


\section{TD/TC/WP(2003)40/FINAL}

corriger des dysfonctionnements du marché et réaliser des objectifs non économiques, tels que la protection des consommateurs. Comme l'AGCS le reconnaît lui-même, la libéralisation ne consiste pas à abolir toutes les réglementations. De fait, les marchés libéralisés ont souvent besoin d'être plus réglementés que moins. La question est toutefois de savoir dans quelle mesure la réalisation de ces objectifs d'ordre public est nécessairement garantie par les dispositions prises et si l'on ne pourrait pas l'obtenir aussi bien ou mieux par des mesures moins restrictives pour les échanges.

44. La raison d'être des prescriptions concernant la nationalité, par exemple, n'est pas claire. Ces mesures - on l'a vu plus haut - servent à garantir que les juristes diplômés connaissent bien le droit et la culture du pays d'accueil. Poser que la capacité d'atteindre le niveau de connaissances requis pour pouvoir être diplômé est fonction de la nationalité paraît difficilement justifiable. Si un étranger est prêt à faire ce qu'il faut pour acquérir la connaissance requise du pays considéré ou montrer qu'il l'a (faire toutes ses études juridiques dans le pays, obtenir les diplômes locaux voulus, par exemple) les consommateurs seront aussi bien protégés sans qu'il soit besoin d'une prescription concernant la nationalité $e^{53}$. De même, il ne semble pas que le fait qu'un juriste localement qualifié fournisse la totalité des services touchant le droit national pour le compte d'un cabinet de consultations juridiques étrangers présente un risque particulier du point de vue de la protection des consommateurs.

45. Compte tenu des techniques modernes d'information et de communication, peut-être faudra-t-il aussi revoir les assertions selon lesquelles des prescriptions concernant la résidence sont nécessaires pour que les hommes de loi soient proches de leurs clients. On pourrait aussi les évaluer en fonction du fait qu'en règle générale, un cabinet de consultations juridiques ou un homme de loi étranger souhaite par luimême établir des contacts étroits avec ses clients afin d'afin d'avoir une chance de soutenir la concurrence dans le pays d'accueil. Par ailleurs, lorsque des prescriptions concernant la résidence sont justifiées par la protection des consommateurs, il faudra se demander jusqu'à quel point elles sont effectivement nécessaires du point de vue des consommateurs. D'ailleurs, comme indiqué précédemment, la demande d'échanges internationaux de services juridiques émane essentiellement d'entreprises commerciales. Il y a tout lieu de penser que ces consommateurs avertis sont capables de se protéger plus efficacement par des accords contractuels privés - contenant des clauses sur l'assurance, l'indemnisation et le règlement des différends - que par ce que cherchent à leur procurer des prescriptions concernant la résidence ${ }^{54}$.

46. Des mesures de réglementation trop restrictives peuvent créer des distorsions qui finissent par marginaliser purement et simplement le secteur national et freiner sa croissance et celle de l'économie nationale. Limiter la possibilité, pour les hommes de loi locaux, de former des partenariats avec des juristes étrangers ou de faire appel à leurs services risque, par exemple, d'avoir pour unique conséquence que ce seront les juristes étrangers qui accompliront tout le travail lucratif dans le domaine du commerce international, sans même un transfert de connaissances aux juristes locaux. Les règles qui soumettent la création de filiales de cabinets de consultations juridiques étrangers à des procédures longues et rigoureuses risquent de se limiter à ralentir les investissements étrangers et la création de cabinets locaux compétitifs à l'échelle internationale ${ }^{55}$.

47. Pour tirer pleinement parti de la libéralisation et veiller à préserver les principaux objectifs d'ordre public, il faut, au contraire, une réglementation mieux adaptée et plus ciblée. L'amélioration, par exemple, de la connaissance des règles et de la culture locales est, semble-t-il, mieux assurée par un processus transparent, objectif et équitable conférant le droit de pratiquer le droit local sur la base du savoir, des compétences et de l'aptitude professionnelle que par des prescriptions concernant la

\footnotetext{
53 OCDE, 1997.

54 OCDE, 1997.

55

Hook, 2003.
} 
nationalitée ${ }^{56}$. De même, on peut répondre au besoin de proximité et à la nécessité de protéger les consommateurs par d'autres mesures, moins restrictives pour le commerce, que des prescriptions concernant la résidence: collaboration avec les nationaux, obligation de souscrire à une obligation financière afin de garantir la possibilité de recours ou souscription d'une assurance-responsabilité ${ }^{57}$. En outre, on peut remédier à la crainte que la constitution en société n'entraîne une dilution de la responsabilité professionnelle en autorisant des partenariats qui préservent intégralement la responsabilité individuelle des praticiens envers leurs clients ${ }^{58}$.

48. On pourrait même aller plus loin et créer des cadres réglementaires qui, non seulement préserveraient les objectifs traditionnels, mais stimuleraient effectivement le transfert de connaissances et de compétences, par exemple, en demandant (et non en évitant) une coopération entre les cabinets nationaux et étrangers ou la formation des hommes de loi locaux. C'est seulement par une coopération directe de cet ordre que les cabinets locaux pourront parvenir au niveau qualitatif indispensable pour pouvoir être compétitifs à l'échelle internationale ${ }^{59}$.

49. Outre la nécessité que la réglementation corrige les dysfonctionnements du marché et permette d'atteindre les objectifs non économiques et sociaux, certains pays perçoivent la libéralisation comme une menace pour leur propre secteur des services juridiques et estiment qu'un certain degré de protection est nécessaire à cet effet ${ }^{60}$. L'opposition des bureaux de consultation déjà en place et des travailleurs affectés, qui verront souvent dans la libéralisation une menace pour l'emploi ou la rentabilité, ne fait qu'ajouter à la complexité des efforts requis pour libéraliser ce secteur.

50. La libéralisation du commerce s'effectue rarement sans répercussions sur la distribution. Un grand nombre des avantages découlant d'une plus grande ouverture du marché exigeront un certain temps avant de se manifester, alors que les coûts liés à la rationalisation du secteur apparaîtront au contraire rapidement avec des modifications de la structure de la propriété et d'éventuels déplacements de maind'œuvre. Il importe toutefois de reconnaître que, comme indiqué dans la section précédente, la libéralisation expose aussi l'économie nationale à des niveaux plus élevés de concurrence, d'investissement étranger et d'efficience générale. Ces avantages pourraient bien compenser les pertes subies par le secteur juridique aux intérêts concurrents.

51. Un aspect important de la libéralisation des échanges et de l'investissement dans le secteur des services juridiques, comme dans bien d'autres branches des services, est que l'ajustement associé à une plus grande ouverture des marchés a généralement lieu dans un climat sectoriel dynamique, où les entreprises en expansion peuvent plus facilement absorber les travailleurs touchés. Ceux qui risquent le plus de souffrir de cette évolution trouveront peut-être plus facile de se repositionner dans un secteur dynamique de nature à créer de bonnes possibilités de revenu et d'emploi que dans un système stagnant réfractaire au changement. En outre, une caractéristique particulière des services juridiques est que les fournisseurs

56 OMC, 2001a.

57 OMC, $2001 \mathrm{~b}$.

58 OCDE, 1997. Dans le cadre élargi des services professionnels, on a examiné à l'OCDE les moyens de faire progresser la réforme de la réglementation. On a notamment recherché des solutions autres, moins contraignantes/fastidieuses, que des restrictions à l'investissement dans quatre secteurs des services professionnels (services comptables, services juridiques, services architecturaux et services d'ingénierie). Dans bien des cas, les discussions se sont appuyées sur des comparaisons entre ces quatre domaines et sur l'expérience positive des pays membres de l'OCDE qui ouvrent davantage leur marché aux services professionnels tout en assurant une protection adéquate des consommateurs et des intérêts publics. Par la suite, le Conseil a approuvé ces autres approches de la réglementation [voir OCDE (2003b)].

Hook, 2003; et Department for Constitutional Affairs, Royaume-Uni, 2003a.

60

Le reste de cette section est, pour l'essentiel, fondé sur OCDE, 2002b. 


\section{TD/TC/WP(2003)40/FINAL}

nationaux conservent un avantage compétitif dans la pratique du droit du pays d'accueil, si bien que l'ajustement associé à une ouverture accrue du marché s'y fait généralement plus facilement que dans d'autres secteurs des services. C'est aussi vrai en raison du fait que les cabinets de consultations juridiques étrangers ont tendance à engager des hommes de loi locaux pour développer leurs activités dans le domaine du droit du pays d'accueil.

\section{ii) Le cadre de négociation de l'AGCS}

52. Une libéralisation menée de manière progressive, ordonnée et transparente peut non seulement aider à obtenir les avantages susmentionnés, mais permettre aux entreprises en place de se préparer à une concurrence plus vive et aux pouvoirs publics de veiller à mettre en place le cadre réglementaire approprié pour pour régir la nouvelle situation concurrentielle du marché.

53. L'AGCS est particulièrement bien adapté à la réalisation de ces objectifs : il donne, en effet, aux Membres de l'OMC toute liberté de choisir les conditions auxquelles l'ouverture du marché pourra se faire. Dans le cadre des négociations actuelles fondées sur le principe des offres et des requêtes, ces Membres se trouvent placés devant plusieurs possibilités. Lorsque les Membres choisissent volontairement d'inscrire sur leurs listes un engagement concernant le secteur des services juridiques, par exemple, ils peuvent, notamment :

- exclure certaines parties du secteur (la pratique du droit du pays d'accueil, par exemple), un soussecteur (la représentation devant les tribunaux, par exemple) ou un mode particulier de fourniture.

- limiter l'accès des fournisseurs étrangers, ou opérer une discrimination à l'encontre de ceux-ci, dans le secteur, dans un sous-secteur ou pour un mode de fourniture en inscrivant sur leurs listes les mesures relatives à l'accès au marché et au traitement national. Comme indiqué dans la section précédente, il convient toutefois de veiller à réformer la réglementation de façon à ce que les objectifs d'ordre public, tels que la protection des consommateurs, continuent d'être assurés de manière efficace.

- dans le cas des pays en développement, ajouter les qualifications à leurs listes d'engagements spécifiques afin de renforcer leurs capacités nationales dans le domaine des services, notamment grâce à un accès aux technologies et au savoir-faire. Le cadre de l'AGCS offre aux pays en développement suffisamment de souplesse pour poursuivre ces objectifs, en particulier grâce aux dispositions des articles IV et XIX. En exploitant ces qualifications, il conviendra toutefois de veiller à ce qu'elles ne finissent pas par avoir un effet dissuasif sur les échanges et l'investissement, ce qui retarderait l'expansion des capacités nationales.

- s'engager préalablement à opérer une libéralisation dans l'avenir afin de donner à l'industrie locale le temps de s'ajuster ou un délai supplémentaire pour faire en sorte que le cadre réglementaire approprié ait été mis en place.

- appliquer toutes mesures de réglementation intérieure non discriminatoires, telles que l'assurance-responsabilité, sans obligation de les inscrire sur les listes. Ces mesures tombent sous le coup de l'article VI de l'AGCS. ${ }^{61}$

54. Une question capitale pour de nombreux pays en développement est celle de la capacité de réglementation, c'est-à-dire celle de savoir si ces pays disposent des ressources humaines et

61 Néanmoins, la CE a suggéré dans sa communication relative à la classification qu'il pourrait être utile d'en faire état dans une note. 
institutionnelles requises pour concevoir, administrer et appliquer le cadre réglementaire indispensable au succès de la libéralisation. Une évaluation de cette capacité devra déterminer la nature et le rythme de cette dernière. L'octroi aux pays en développement d'une assistance technique visant à les doter de capacités de réglementation sera donc un élément important des négociations relatives à l'AGCS.

\section{Réglementation intérieure ${ }^{62}$}

\section{i) Prescriptions concernant les qualifications}

55. L'abolition ou l'assouplissement des mesures concernant l'accès au marché et le traitement national, bien qu'essentiels, peuvent ne pas suffire pour ouvrir largement le marché au secteur juridique. Les prescriptions en matière de qualifications, certes légitimes, peuvent influer profondément sur les échanges de services juridiques, notamment en ce qui concerne la pratique du droit du pays d'accueil. La formation juridique varie d'un pays à l'autre et, dans certains cas, les écarts sont tels que les juristes étrangers sont obligés d'acquérir de nouvelles qualifications pour pouvoir exercer dans le pays d'accueil. Dans la plupart des pays, les qualifications juridiques requises comprennent un diplôme universitaire sanctionnant entre trois et cinq ans d'études (complétées, dans certains cas, par des études du troisième cycle) et un stage pratique suivi d'un examen d'aptitude professionnelle ${ }^{63}$. Qui plus est, les aspects procéduraux de ces prescriptions, tels que le traitement des demandes ou la fréquence des examens, peuvent constituer des obstacles aux échanges dans ce secteur.

56. Les accords de reconnaissance mutuelle (ARM) peuvent servir à éliminer ou à réduire au maximum ces différences et contribuer à éviter d'éventuels doubles emplois dans les prescriptions concernant l'accréditation ou l'attribution de licences. Ce mécanisme de réglementation peut être très prometteur dans le contexte des services juridiques. L'Union européenne a acquis une grande expérience de l'harmonisation et de la reconnaissance mutuelle des règles juridiques dans le cadre de sa mise en place d'un marché intérieur unique (voir encadré 2). Les questions qui se posent sont les équivalences entre différents programmes d'enseignement et prescriptions en matière de formation; les domaines du droit devant être couverts; et les moyens spécifiques par lesquels les praticiens peuvent être tenus responsables de pratiques contraires à la profession à l'échelle internationale. ${ }^{64}$

62 Cette section porte sur les questions générales de qualifications i) et sur les prescriptions concernant les licences pour les consultants juridiques étrangers ii). Un autre aspect de la réglementation intérieure qui est sujet à controverse est le traitement des pratiques multidisciplinaires. Beaucoup de pays interdisent l'association entre juristes et non-juristes pour des raisons liées à la nécessité d'assurer la protection du consommateur et la qualité des services. Voir OMC, 1998a.

OMC, 1998a.

64

OCDE, 2002a. 
TD/TC/WP(2003)40/FINAL

\section{Encadré 2. Reconnaissance mutuelle et établissement dans l'Union européenne}

L'UE concevait auparavant la reconnaissance comme la reconnaissance de l'expérience (trois à six ans normalement) et des diplômes professionnels, sur la base de systèmes visant à harmoniser globalement l'enseignement et de la formation. Cette approche s'est toutefois révélée trop lourde et trop complexe et elle a fait place à une nouvelle démarche axée sur la reconnaissance mutuelle. L'harmonisation des conditions d'accès aux professions a été remplacée par un système général de reconnaissance des diplômes de l'enseignement supérieur en vertu duquel les États membres reconnaissent la comparabilité de ces diplômes notamment pour accorder l'autorisation d'exercer une profession réglementée. Le système actuel inclut donc à la fois des directives sectorielles couvrant des professions particulières et une approche plus générale couvrant les professions réglementées qui ne font pas l'objet de directives spécifiques. Le système communautaire de reconnaissance mutuelle est destiné à faciliter la liberté de circulation des ressortissants de l'UE et ne s'étend pas aux ressortissants de pays tiers.

Un système général de reconnaissance existe pour les professions qui ne sont pas couvertes par des directives spécifiques. La directive 89/48/CEE a mis en place un premier système général de reconnaissance des diplômes de l'enseignement supérieur sanctionnant des formations professionnelles d'au moins trois ans. La directive 92/51/CEE a étendu ce système aux diplômes, certificats et titres ne relevant pas de l'enseignement supérieur de longue durée, en couvrant à la fois les cycles courts d'études post-secondaires ou professionnelles et les cycles d'études secondaires. Enfin, la directive 99/42/CE a introduit un système de reconnaissance mutuelle des qualifications pour l'accès à certaines activités commerciales, industrielles ou artisanales qui n'étaient pas encore couvertes par les directives 89/48/CEE et 92/51/CEE, en annulant en même temps les directives sectorielles dans ce domaine particulier.

Dans les trois cas, l'État membre d'accueil ne peut refuser l'accès à l'activité considérée si le demandeur possède la qualification requise dans son pays d'origine. C'est une forme de reconnaissance semi-automatique reposant sur le principe que la formation doit être reconnue lorsque les activités professionnelles réglementées que la personne souhaite exercer sont les mêmes que celles qu'elle est autorisée à exercer, ou qu'elle a déjà exercées, dans son pays d'origine et lorsqu'il n'existe pas de différence fondamentale entre les qualifications requises dans le pays d'accueil et celles que possède le demandeur. Si la formation reçue est d'une durée inférieure à celle qu'elle est dans le pays d'accueil, ce dernier peut exiger en plus une expérience professionnelle d'une certaine durée. Si la formation est très différente, le pays d'accueil peut imposer au demandeur de suivre un stage d'adaptation ou de subir une épreuve d'aptitude.

Les services juridiques, qui sont couverts par le système général de reconnaissance, ont également fait l'objet de directives sectorielles pour résoudre les problèmes posés par la diversité des systèmes juridiques existant au sein de I'UE. La directive 77/249/CEE fixe les règles de la libre prestation de services juridiques sous le titre professionnel de l'État d'origine et, plus récemment, la directive 98/5/CE a élaboré le cadre réglementaire de l'établissement des avocats, ressortissants de pays de l'UE, dans les différents États membres. Cette directive sur l'établissement permet aux avocats d'un État membre de l'UE d'exercer en permanence sous leur titre professionnel d'origine dans un autre État membre en précisant toutefois que le pays d'accueil peut les obliger à être assistés d'un avocat local lorsqu'ils représentent et défendent leurs clients en justice. Après avoir travaillé pendant trois années sur cette base, ils acquièrent le droit d'exercer pleinement leur profession sous le titre professionnel du pays d'accueil sans avoir à passer un examen d'aptitude.

La Commission européenne a présenté récemment un projet de nouvelle directive ${ }^{65}$ qui vise à rendre le système de reconnaissance mutuelle plus clair et plus facile à comprendre. La nouvelle directive unique proposée réviserait totalement l'ensemble des directives reposant sur la reconnaissance des titres pour maintenir les principales conditions et garanties que ces textes prévoient tout en simplifiant la structure du système et en apportant des améliorations à son fonctionnement. La proposition s'appliquerait aux services juridiques relatifs à toute amélioration apportée au système général de reconnaissance (directives 89/48/CEE et 92/51/CEE). Elle simplifierait aussi la reconnaissance pour un certain nombre de professions particulières (médecins, infirmiers responsables des soins généraux, praticiens de l'art dentaire, vétérinaires, sages-femmes, pharmaciens et architectes), sans toutefois comprendre les services juridiques.

Source : OCDE, 2002c. 
57. À l'heure actuelle, la plupart des ARM ont néanmoins été conclus entre des économies développées, même si des travaux ont été entrepris pour lier pays développés et pays en développement (dans le cadre de l'ALENA et de l'APEC, par exemple). La plupart des accords commerciaux délèguent l'élaboration des accords de reconnaissance à des organismes professionnels. L'expérience acquise en matière d'ARM tend à prouver que les progrès les plus importants surviennent lorsque l'initiative est lancée par la profession elle-même ${ }^{66}$.

58. L'AGCS permet la conclusion d'ARM dans son article VII (Reconnaissance) qui prévoit que les Membres peuvent reconnaître l'éducation ou l'expérience acquise dans un pays membre de l'OMC, mais non dans un autre (ce qui constitue une exception au traitement NPF). De manière plus générale, les prescriptions en matière de qualifications relèvent de l'article VI de l'AGCS, aux termes duquel le Conseil du commerce des services élabore toutes disciplines multilatérales requises pour faire en sorte que ces prescriptions, celles concernant les licences et celles concernant les normes techniques ne constituent pas des obstacles non nécessaires au commerce des services ${ }^{67}$.

59. La première mesure prise par les Membres de l'OMC en vertu de ce mandat a été l'élaboration en 1998 de disciplines sur la réglementation intérieure dans le secteur de la comptabilité. Ces disciplines, qui ne sont pas encore entrées en vigueur, portent sur les prescriptions relatives aux qualifications, les procédures d'attribution des licences et les normes techniques dans le secteur comptable et des discussions se poursuivent en vue d'étudier la possibilité de les étendre au secteur juridique. Dans le cadre du programme d'activité du Groupe de travail de la réglementation intérieure, plusieurs Membres de l'OMC ont consulté des organes gouvernementaux et des associations professionnelles locales sur la possibilité d'appliquer les disciplines comptables à d'autres services professionnels ${ }^{68}$. Il en ressort que, dans la plupart des cas, les disciplines relatives à la comptabilité peuvent convenir et qu'elles s'appliquent de manière générale à d'autres professions, dont les services juridiques. Il en ressort aussi qu'il faut néanmoins tenir dûment compte des caractéristiques et des conditions d'exercice propres à la profession juridique, de même que de la nécessité de protéger le consommateur et la qualité des services ${ }^{69}$.

\section{ii) Consultants juridiques étrangers}

60. Dans les domaines du droit du pays d'origine/d'un pays tiers et du droit international, les prescriptions concernant les qualifications présentent moins d'importance que dans le domaine du droit du pays d'accueil. Les consultants ou conseils juridiques étrangers, rencontrent moins d'obstacles au

66 OCDE, 2002c.

67 L'article VI énonce déjà quelques disciplines de base visant à limiter les effets restrictifs que les règlements intérieurs peuvent avoir sur le commerce. En particulier, dans les secteurs où des engagements spécifiques concernant des services professionnels sont contractés, les Membres doivent prévoir des procédures adéquates pour vérifier la compétence des professionnels de tout autre Membre. De manière plus générale, dans les secteurs où des engagements spécifiques sont contractés, les règlements doivent être administrés d'une manière raisonnable, objective et impartiale.

Jusqu'à ce jour, 11 Membres ont soumis des communications préliminaires sur le résultat des consultations : Canada (deux propositions: OMC, 2001f; et OMC, 2003b), Chine (OMC, 2002b), Territoire douanier distinct de Taïwan, Penghu, Kinmen et Matsu (OMC, 2003c), les Communautés européennes et leurs États membres (OMC, 2000d), Hong Kong, Chine (OMC, 2000e), Japon (OMC, 2000f), République de Corée (OMC, 2000g), Mexique (OMC, 2001g), Pologne (OMC, 2000h), Suisse (OMC, 2001h) et Thailande (OMC, 2001i).

L'Association internationale du barreau a aussi contribué au débat en rédigeant un document de travail intitulé: "What Changes are required to WTO Accountancy Disciplines before they can be applied to the Legal Profession?" (IBA, 2003b). Les modifications qu'il est proposé d'apporter aux disciplines relatives aux services comptables comprennent, entre autres, la nécessité d'ajouter quelques précisions sur les valeurs essentielles de la profession juridique et de clarifier la distinction entre les prescriptions concernant les qualifications et celles qui concernent les licences. 
commerce des services; ils peuvent néanmoins se heurter à des prescriptions réglementaires de taille, notamment en ce qui concerne les licences. La plupart des pays, par exemple, ne permettent pas aux consultants de se présenter en tant que membres de la profession locale, mais exigent qu'ils utilisent un titre qui leur est propre ou leur titre professionnel d'origine, afin d'être différenciés des avocats locaux. Dans certains pays, les consultants sont tenus de s'inscrire au barreau local et/ou de réussir un examen d'aptitude professionnelle. Même si ces examens sont souvent moins onéreux qu'une formation locale complète, ils peuvent représenter un obstacle, surtout s'ils ont lieu dans la langue locale. Plusieurs pays exigent aussi que les consultants juridiques étrangers aient un certain nombre d'années de pratique dans leur pays d'origine afin de pouvoir exercer dans le pays d'accueil.

61. Depuis quelques années, on travaille à l'échelle tant nationale qu'internationale à assouplir ces prescriptions tout en préservant l'intérêt général. La notion de consultant juridique a été adoptée pour la première fois par l'État de New York en 1974. Les hommes de loi étrangers peuvent être agréés en tant que consultants juridiques sans examen (c'est-à-dire sans être obligés d'avoir les qualifications requises pour être membres du barreau de New York) pour pratiquer le droit de leur pays d'origine ou d'un pays tiers, ou le droit international dans cet État, et pour fournir des conseils sur la plupart des domaines du droit des États-Unis s'ils travaillent avec un avocat américain. La condition à cet effet est qu'ils aient une certaine expérience pratique et acceptent de s'abstenir d'exercer dans certains domaines du droit local ${ }^{70}$. En 1993, l'American Bar Association ( $\mathrm{ABA}$ ) a publié un Règlement type (des lignes directrices) à l'intention des consultants juridiques étrangers qui ressemble étroitement aux règles initialement adoptées par l'État de New York. L'objet de cette mesure est de tenter de rationaliser les procédures d'admission des consultants juridiques étrangers et d'instaurer une plus grande uniformité et davantage de clarté d'un bout à l'autre des États-Unis. A ce jour, 23 États et le District de Columbia ont adopté des règles similaires.

62. Les accords régionaux prévoient aussi des règles touchant les consultants juridiques étrangers. La notion de consultant juridique, par exemple, est inhérente à la législation communautaire sur les services juridiques décrite dans l'encadré 2. En outre, les parties à l'ALENA sont tenues de faire en sorte que les consultants juridiques étrangers d'autres États membres soient autorisés à exercer ou à conseiller sur le droit du pays dans lequel ils sont compétents pour exercer leur métier. L'Accord contient aussi des clauses relatives à la libéralisation future et des dispositions prévoyant l'obligation d'organiser des consultations avec les organes professionnels sur des questions telles que les partenariats entre hommes de loi autorisés à exercer localement et consultants juridiques étrangers, et les normes relatives à l'autorisation d'exercer de ces derniers ${ }^{71}$. On trouve aussi des dispositions similaires dans des accords régionaux plus récents, comme l'Accord de libre-échange entre les États-Unis et le Chili.

63. L'Association internationale du barreau (IBA) a aussi contribué à clarifier et à uniformiser les règles internationales relatives aux avocats étrangers. Elle a adopté en 1998 une déclaration intitulée "Statement of General Principles for the Establishment and Regulation of Foreign Lawyers"72 (Principes généraux régissant l'établissement et la réglementation des activités des avocats étrangers), qui énonce des règles générales touchant l'établissement des avocats étrangers, y compris des consultants juridiques. Elle définit aussi deux grandes approches de l'attitude à l'égard de l'autorisation d'exercer de ces avocats et de la réglementation de leur activité : une approche prévoyant la nécessité de posséder toutes les qualifications du pays d'accueil, mais aucune restriction quant au domaine d'activité, et une approche interdisant aux avocats étrangers de pratiquer le droit du pays d'accueil mais n'exigeant de leur part aucune qualification de ce pays.

\footnotetext{
70 Plaidoiries devant les tribunaux de l'État, droit foncier, droit familial et établissement des documents régissant les successions. Voir Cone, 2003 ; et Silver, à paraître.

72

IBA, 1998.
} 
64. Dans le cadre des négociations en cours de l'OMC, l'Australie (où plusieurs juridictions ont adopté des règles conformes à la déclaration de l'IBA) défend l'idée que les fournisseurs de services juridiques n'ont pas besoin d'autorisation pour fournir des services portant sur tous les aspects du droit d'une ou de plusieurs juridictions, mais à la condition expresse de posséder les compétences ou les qualifications voulues. Par suite, les avocats étrangers souhaitant fournir des services juridiques relatifs à leur pays d'origine ou à un pays tiers ne seraient pas tenus d'obtenir une autorisation complète en se lançant dans le processus souvent laborieux de se requalifier complètement dans le pays d'accueil. Ces dispositions pourraient aussi comporter des partenariats entre praticiens étrangers et praticiens du pays d'accueil, de façon à ce que les clients demandant des services juridiques internationaux puissent obtenir une large gamme de services d'un prestataire commun à plusieurs juridictions ${ }^{73}$.

65. Dans les négociations en cours fondées sur les offres et les requêtes, les Membres qui souhaitent prendre des engagements relatifs aux services juridiques peuvent le faire en définissant la portée sectorielle par référence au concept de consultants juridiques étrangers. En le faisant, ils peuvent aussi préciser dans leurs offres, notamment, les domaines du droit (droit international, droit du pays d'origine, droit d'un pays tiers) dans lesquels les consultants juridiques étrangers peuvent être autorisés à exercer, les prescriptions qui leur sont applicables en matière d'expérience préalable ou le fait qu'ils sont ou non autorisés à former des partenariats avec des avocats locaux. Il a aussi été suggéré de combiner les engagements limités aux consultants juridiques étrangers à d'autres engagements fondés sur un document de référence à établir, ce qui pourrait atténuer les problèmes rencontrés par les consultants qui fournissent des services internationaux à leurs clients. Ces engagements porteraient, par exemple, sur des questions de procédure et d'ordre administratif, comme l'enregistrement des établissements, le traitement des demandes dans des délais raisonnables et la transparence. Une autre proposition encore a été de prendre des engagements sur tous les services juridiques sans distinction, en gardant à l'esprit que, quelle que soit l'ampleur des engagements, la fourniture de services juridiques concernant le droit du pays d'accueil restera réservée aux juristes qualifiés en la matière (sauf si les prescriptions en matière de qualifications sont expressément levées). Ces engagements engloberaient le concept de consultants juridiques étrangers et la possibilité pour les hommes de loi et les cabinets de consultations juridiques étrangers de conclure des partenariats avec des juristes qualifiés du pays d'accueil ou d'en embaucher. ${ }^{74}$

\section{Transparence}

66. La transparence du processus de réglementation est fondamentale pour la compétitivité du marché des services juridiques. Cette remarque vaut particulièrement pour le commerce des services juridiques, attendu que les mesures qui influent sur l'entrée sur le marché des services juridiques et sur le fonctionnement de celui-ci comprennent toutes sortes de lois, de règlements et de pratiques en vigueur au plan national. Il est indispensable que ces mesures puissent être facilement consultées aussi bien par le public - particuliers et entreprises - que par les fournisseurs de services juridiques.

67. Dans l'AGCS, des dispositions relatives à la transparence sont énoncées à l'article III, aux termes duquel les Membres de l'OMC publient toutes les mesures d'application générale pertinentes, informent le Conseil du commerce des services de l'adoption des nouvelles lois et réglementations, et des modifications des lois et réglementations existantes, dans les secteurs visés par des engagements spécifiques, et

73 OMC, 2001c.

74 Selon les partisans de cette proposition, lorsque les secteurs visés par les engagements comprennent le droit du pays d'accueil, cela revient à reconnaître le droit des hommes de loi et des cabinets de consultations juridiques étrangers de conclure des partenariats avec des juristes qualifiés du pays d'accueil ou d'en embaucher, sauf si cela est expressément exclu par une limitation de l'accès aux marchés. A l'inverse, lorsque le champ couvert par les engagements ne comprend pas l'exercice du droit du pays d'accueil, cette possibilité nécessiterait un engagement à faire figurer dans la colonne des engagements supplémentaires. C'est que - fait-on valoir - par définition, tout engagement ou limitation au titre des colonnes « accès aux marchés » et « traitement national » ne peut, dans le deuxième cas, influer sur l'exercice du droit du pays d'accueil. 


\section{TD/TC/WP(2003)40/FINAL}

établissent des points d'information. Dans le cadre de la réglementation intérieure, les Membres de l'OMC sont aussi convenus d'adopter de nouvelles disciplines sur la transparence pour le secteur comptable, qui pourraient aussi s'appliquer au secteur juridique. Elles-ci visent en particulier à accroître la transparence des procédures de qualification et d'attribution des licences, ainsi que celle des normes techniques.

68. Les disciplines relatives à la transparence qui dépassent la portée de l'article III font partie du programme de travail visant à élaborer des disciplines horizontales sur la réglementation intérieure. Plusieurs Membres ${ }^{75}$ ont soumis des propositions dans le but de renforcer les dispositions de cet article III en y ajoutant des prescriptions supplémentaires. Ces propositions ont en commun le point de vue selon lequel de nouvelles règles pourraient être établies pour accroître la transparence des prescriptions et des procédures en matière de licences, ainsi que celle des normes techniques, conformément aux dispositions que contiennent déjà les disciplines relatives aux services comptables.

69. Les avis des Membres sont toutefois plus partagés sur la question de savoir s'il conviendrait aussi d'élaborer des règles sur l'obligation de tenir des consultations préalables. Si certains sont favorables à l'établissement de telles règles, d'autres estiment qu'elles risqueraient d'être incompatibles avec leurs systèmes réglementaire et législatif ou qu'elles représenteraient une lourde charge administrative, notamment pour les pays peu développés. À ce propos, les dispositions figurant dans les disciplines comptables, qui reconnaissent les difficultés que peut soulever la consultation préalable et demandent aux Membres de s'efforcer de donner la possibilité de formuler des observations lorsqu'ils introduisent de nouvelles mesures, pourraient contribuer de façon précieuse aux travaux en cours sur des disciplines en matière de réglementation.

75 Australie (deux propositions : OMC, 1999; et OMC, 2000i), les Communautés européennes et leurs États membres (deux propositions : OMC, 2001j; et OMC 2003d); et États-Unis (OMC, 2000j). 


\section{TABLEAU 1 DE L'ANNEXE LISTES ÉTABLIES EN VUE DES NÉGOCIATIONS ${ }^{76}$}

\section{Questions relatives à l'AGCS}

\begin{abstract}
a) Mesures affectant les prestations transfrontalières, y compris la consommation à l'étranger
\end{abstract}

1. Les fournisseurs de services juridiques non résidents peuvent-ils desservir le marché sur une base transfrontalière?

2. Quels sont les domaines du droit où les prestations transfrontalières sont autorisées et ceux où elles sont limitées?

3. La transmission électronique de services juridiques par des fournisseurs de services juridiques étrangers non établis est-elle soumise à restrictions?

4. Où ces restrictions sont-elles énoncées et le sont-elles clairement?

5. Quelles sont les raisons de politique générale justifiant ces restrictions (garantir la connaissance des règles locales ou le respect de la responsabilité à l'égard du public, par exemple)?

6. Peut-on, pour ce faire, utiliser d'autres moyens moins restrictifs pour les échanges (une assurance responsabilité, par exemple)?

b) Mesures affectant la présence commerciale et le mouvement des personnes physiques

\section{Questions propres à un secteur}

1. Existe-t-il des prescriptions concernant la nationalité?Dans l'affirmative, s'appliquent-elles uniquement à la pratique du droit du pays d'accueil? Les cabinets et juristes étrangers souhaitant exercer le droit de leur pays d'origine, le droit d'un pays tiers (dans lequel ils sont qualifiés) ou le droit international sont-ils autorisés à le faire malgré les prescriptions concernant la nationalité?

2. Quelle est la définition du droit du pays d'origine? Cette définition est-elle par trop restrictive?

3. Les fournisseurs étrangers sont-ils tenus de s'établir localement selon une forme juridique particulière? Dans l'affirmative, quelle est-elle/quelles sontelles?

4. Des prescriptions concernant la résidence préalable s'appliquent-elles aux cabinets et aux praticiens étrangers? Les praticiens étrangers sont-ils tenus d'obtenir un droit de résidence permanente ?

5. Les cabinets de consultations juridiques étrangers peuvent-ils former des partenariats avec des professionnels locaux? Peuvent-ils engager des professionnels titulaires d'un diplôme local?

6. Des prescriptions en matière de personnel s'appliquent-elles à l'établissement de filiales: nombre de partenaires ou d'avocats par bureau, ou proportion d'avocats locaux par rapport aux avocats étrangers, par exemple?

7. Existe-t-il des restrictions à l'utilisation des raisons sociales de cabinets étrangers internationaux?

8. Où et avec quelle précision ces restrictions sont-elles énoncées ?

9. Quelles sont les raisons justifiant ces restrictions (garantir la connaissance des règles locales, la compétence, la proximité et la transparence, par exemple)?

10. Est-il possible de satisfaire à ce critère par d'autres moyens moins restrictifs pour le commerce (prescriptions objectives prévoyant un contrôle fondé sur la compétence et des dispositions relatives à la pratique locale, désignation d'un représentant ou souscription d'une assurance responsabilité, par exemple)?

76 Les listes ci-après peuvent être utilisées aux fins suivantes : élaborer l'offre d'un Membre de l'OMC ; évaluer une requête d'un partenaire commercial d'un Membre; évaluer une offre d'un partenaire commercial d'un Membre ; formuler une requête ; ou décider de cautionner ou non une position adoptée par un autre Membre de l'OMC. 


\begin{tabular}{|c|c|}
\hline & $\begin{array}{l}\text { Mesures horizontales } \\
\text { 1. Existe-t-il des restrictions concernant le capital étranger? } \\
\text { 2. Existe-t-il des restrictions au mouvement du personnel professionnel, } \\
\text { administratif et technique? Et aux transferts de personnel d'une filiale à une } \\
\text { autre d'une même entreprise? Ces restrictions s'appliquent-elles aux personnes } \\
\text { physiques cherchant à s'établir durablement ou aux personnes n'effectuant que } \\
\text { de brefs voyages d'affaires? } \\
\text { 3. Dans le cas des fournisseurs contractuels de services, les mêmes restrictions } \\
\text { s'appliquent-elles aux salariés des cabinets de consultations juridiques et aux } \\
\text { hommes de loi indépendants? } \\
\text { 4. L'octroi d'un visa est-il subordonné à l'obligation d'avoir une expérience } \\
\text { préalable ou d'avoir eu une expérience après l'obtention de la qualification? }\end{array}$ \\
\hline $\begin{array}{l}\text { c) Mesures relatives à } \\
\text { l'attribution des licences et } \\
\text { à la réglementation } \\
\text { intérieure }^{77}\end{array}$ & $\begin{array}{l}\text { Considérations générales } \\
\text { 1. Quels sont les lois et règlements qui régissent l'autorisation de fournir des } \\
\text { services juridiques? } \\
\text { 2. Par qui les autorisations sont-elles accordées et contrôlées ? } \\
\text { 3. Des autorisations sont-elles requises pour les cabinets nationaux ou étrangers } \\
\text { (ou pour les deux)? } \\
\text { 4. Pour l'obtention d'une autorisation, les fournisseurs de services étrangers sont- } \\
\text { ils soumis à des prescriptions différentes de celles appliquées aux fournisseurs } \\
\text { nationaux ou à des prescriptions supplémentaires? } \\
\text { 5. Une pratique multidisciplinaire est-elle autorisée? } \\
\text { 6. Quelles sont les qualifications requises pour exercer le droit du pays d'accueil? } \\
\text { Combien de temps faut-il avoir exercé localement sous la direction d'un } \\
\text { 7. professionnel du pays avant de pouvoir être qualifié? } \\
\text { 8. Par qualifications étrangères sont-elles reconnues? } \\
\text { de qualification sont-ils organisés? Cette procédure est-elle transparente? } \\
\text { 9. Existe-t-il des restrictions différentes pour les hommes de loi nationaux qui } \\
\text { exercent à l'étranger? }\end{array}$ \\
\hline
\end{tabular}

77 Certaines de ces mesures peuvent être considérées comme limitant l'accès aux marchés ou le traitement national si elles tombent sous le coup des articles XVI et XVII de l'AGCS. 


\begin{tabular}{|c|c|}
\hline & $\begin{array}{l}\text { Consultants juridiques étrangers } \\
\text { 1. Des prescriptions en matière de qualifications s'appliquent-elles à l'exercice du } \\
\text { droit d'un pays étranger ou du droit international ? Dans l'affirmative, existe-t-il } \\
\text { un régime de licences différent ou "limité" pour les praticiens étrangers qui ne } \\
\text { souhaitent exercer que le droit de leur pays d'origine, le droit d'un pays tiers } \\
\text { (pour lequel ils possèdent une qualification) ou le droit international? } \\
\text { 2. Ces praticiens sont-ils tenus d'observer les règles de déontologie du pays } \\
\text { d'accueil? Sont-ils considérés comme des avocats? } \\
\text { 3. Sont-ils autorisés à fournir des services consultatifs sur le droit du pays } \\
\text { d'accueil si ces services s'appuient sur l'avis d'un avocat local pleinement } \\
\text { qualifié? } \\
\text { 4. Peuvent-ils se présenter comme étant des membres de la profession locale ou } \\
\text { doivent-ils utiliser un titre différent? Dans l'affirmative, dans quelle langue? } \\
\text { 5. Ces praticiens doivent-ils être inscrits au barreau local, réussir un examen } \\
\text { d'aptitudes professionnelles, ou les deux? Ledit examen est-il différent de } \\
\text { l'examen local donnant droit à une pleine qualification? Dans quelle langue se } \\
\text { déroule-t-il? } \\
\text { 6es praticiens doivent-ils avoir exercé pendant un certain nombre d'années } \\
\text { dans leur pays d'origine pour être autorisés à exercer dans le pays d'accueil? } \\
\text { Dans l'affirmative, combien d'années d'expérience sont-elles requises? }\end{array}$ \\
\hline $\begin{array}{l}\text { e) Mesures de } \\
\text { libéralisation } \\
\text { préférentielles }\end{array}$ & $\begin{array}{l}\text { 1. Des accords préférentiels influent-ils sur la fourniture de services juridiques? } \\
\text { Quelles sont les mesures qui font l'objet d'un traitement préférentiel? Des } \\
\text { mesures préférentielles s'appliquent-elles aussi au mouvement de personnes } \\
\text { physiques? } \\
\text { 2. Quelles conditions les fournisseurs étrangers doivent-ils remplir pour être en } \\
\text { conformité avec lers règles visées dans les ARM existants auxquels les } \\
\text { fournisseurs des pays d'accueil sont parties? Les fournisseurs de services } \\
\text { étrangers doivent-ils être établis localement pour être habilités à participer à un } \\
\text { ARM? } \\
\text { 3. Le pays importateur applique-t-il des dispositions préférentielles en matière } \\
\text { d'accès aux fournisseurs de services des pays en développement? }\end{array}$ \\
\hline f) Accès universel & $\begin{array}{l}\text { Le gouvernement applique-t-il des obligations en matière d'accès aux services juridiques } \\
\text { ou d'aide juridique gratuite? Comment ces obligations sont-elles définies? }\end{array}$ \\
\hline
\end{tabular}

\section{Questions non liées à l'AGCS}

$$
\text { g) Politique de la }
$$
g) Politique de

1. Existe-t-il dans le pays importateur des exemptions sectorielles à la législation sur la concurrence qui influent sur les conditions de concurrence sur les marchés des services juridiques?

2. Comment la législation sur la concurrence s'applique-t-elle aux cas d'abus de pouvoir de monopole? et aux ententes?

h) Admission temporaire du matériel nécessaire à la fourniture de services
1. Existe-t-il des restrictions à l'admission temporaire du matériel professionnel nécessaire à la fourniture de services sur un marché étranger? 
TABLEAU 2 DE L'ANNEXE

PRINCIPAUX CABINETS DE CONSULTATIONS JURIDIQUES DU MONDE EN 2002

\begin{tabular}{|c|c|c|c|c|c|}
\hline Classement & Nom & Siège social & $\begin{array}{l}\text { Nombre de } \\
\text { juristes }\end{array}$ & $\begin{array}{l}\text { Juristes en } \\
\text { poste hors } \\
\text { du pays } \\
\text { d'origine }\end{array}$ & $\begin{array}{l}\text { Nombre de } \\
\text { pays où le } \\
\text { cabinet a } \\
\text { des } \\
\text { bureaux }\end{array}$ \\
\hline 1 & Clifford Chance & $\begin{array}{l}\text { Royaume-Uni - } \\
\text { International }\end{array}$ & 3322 & $63 \%$ & 19 \\
\hline 2 & Baker \& McKenzie & $\begin{array}{l}\text { Etats-Unis - } \\
\text { International }\end{array}$ & 3094 & $83 \%$ & 37 \\
\hline 3 & $\begin{array}{l}\text { Freshfields Bruckhaus } \\
\text { Deringer }\end{array}$ & $\begin{array}{l}\text { Royaume-Uni - } \\
\text { International }\end{array}$ & 2430 & $61 \%$ & 18 \\
\hline 4 & Allen \& Overy & $\begin{array}{l}\text { Royaume-Uni - } \\
\text { International }\end{array}$ & 2197 & $48 \%$ & 20 \\
\hline 5 & Linklaters & $\begin{array}{l}\text { Royaume-Uni - } \\
\text { International }\end{array}$ & 2000 & $52 \%$ & 22 \\
\hline 6 & Eversheds & $\begin{array}{l}\text { Royaume-Uni - } \\
\text { International }\end{array}$ & 1776 & $4 \%$ & 6 \\
\hline 7 & $\begin{array}{l}\text { Skadden, Arps, Slate, } \\
\text { Meagher \& Flom }\end{array}$ & $\begin{array}{l}\text { Etats-Unis - New } \\
\text { York }\end{array}$ & 1653 & $10 \%$ & 12 \\
\hline 8 & $\begin{array}{l}\text { Jones, Day, Reavis \& } \\
\text { Pogue }\end{array}$ & Etats-Unis - National & 1565 & $18 \%$ & 12 \\
\hline 9 & Lovells & $\begin{array}{l}\text { Royaume-Uni - } \\
\text { International }\end{array}$ & 1432 & $55 \%$ & 15 \\
\hline 10 & White \& Case & $\begin{array}{l}\text { Etats-Unis - } \\
\text { International }\end{array}$ & 1427 & $60 \%$ & 24 \\
\hline 11 & Latham \& Watkins & Etats-Unis - National & 1400 & $17 \%$ & 10 \\
\hline 12 & $\begin{array}{l}\text { Sidley Austin Brown \& } \\
\text { Wood }\end{array}$ & Etats-Unis - National & 1400 & $11 \%$ & 6 \\
\hline 13 & Holland \& Knight & Etats-Unis - National & 1212 & $0 \%$ & 1 \\
\hline 14 & $\begin{array}{l}\text { Mayer, Brown, Rowe \& } \\
\text { Maw }\end{array}$ & Etats-Unis - National & 1197 & $26 \%$ & 5 \\
\hline 15 & Minter Ellison Legal Group & Australie - National & 1139 & $5 \%$ & 6 \\
\hline 16 & Morgan, Lewis \& Bockius & Etats-Unis - National & 1097 & $4 \%$ & 5 \\
\hline 17 & Shearman \& Sterling & $\begin{array}{l}\text { Etats-Unis - New } \\
\text { York }\end{array}$ & 1085 & $37 \%$ & 11 \\
\hline 18 & $\begin{array}{l}\text { Akin, Gump, Strauss, Hauer } \\
\text { \& Feld }\end{array}$ & Etats-Unis - National & 986 & $3 \%$ & 4 \\
\hline 19 & DLA & $\begin{array}{l}\text { Royaume-Uni- } \\
\text { National }\end{array}$ & 984 & $3 \%$ & 4 \\
\hline 20 & Herbert Smith & $\begin{array}{l}\text { Royaume-Uni- } \\
\text { Londres }\end{array}$ & 970 & $26 \%$ & 8 \\
\hline 21 & Foley \& Lardner & $\begin{array}{l}\text { Etats-Unis - } \\
\text { Milwaukee }\end{array}$ & 944 & $0 \%$ & 1 \\
\hline 22 & Garrigues & Espagne - National & 929 & $11 \%$ & 3 \\
\hline 23 & Morrison \& Foerster & $\begin{array}{l}\text { Etats-Unis - San } \\
\text { Francisco }\end{array}$ & 916 & $9 \%$ & 6 \\
\hline 24 & McDermott, Will \& Emery & Etats-Unis - National & 914 & $9 \%$ & 3 \\
\hline 25 & Mallesons Stephen Jaques & Australie - National & 909 & $3 \%$ & 3 \\
\hline 26 & Norton Rose & $\begin{array}{l}\text { Royaume-Uni- } \\
\text { Londres }\end{array}$ & 907 & $33 \%$ & 13 \\
\hline
\end{tabular}


TD/TC/WP(2003)40/FINAL

\begin{tabular}{|c|c|c|c|c|c|}
\hline 27 & Weil, Gotshal \& Manges & $\begin{array}{l}\text { Etats-Unis - - New } \\
\text { York }\end{array}$ & 883 & $21 \%$ & 8 \\
\hline 28 & Denton Wilde Sapte & $\begin{array}{l}\text { Royaume-Uni- } \\
\text { Londres }\end{array}$ & 861 & $36 \%$ & 15 \\
\hline 29 & $\begin{array}{l}\text { Brobeck, Phleger \& } \\
\text { Harrison }\end{array}$ & $\begin{array}{l}\text { Etats-Unis - San } \\
\text { Francisco }\end{array}$ & 834 & $0 \%$ & 1 \\
\hline 30 & Winston \& Strawn & Etats-Unis - Chicago & 825 & $5 \%$ & 3 \\
\hline 31 & Vinson \& Elkins & Etats-Unis - Houston & 814 & $5 \%$ & 5 \\
\hline 32 & Hammond Suddards Edge & $\begin{array}{l}\text { Royaume-Uni- } \\
\text { Londres }\end{array}$ & 805 & $15 \%$ & 7 \\
\hline 33 & Pillsbury Winthrop & $\begin{array}{l}\text { Etats-Unis - San } \\
\text { Francisco }\end{array}$ & 803 & $2 \%$ & 5 \\
\hline 34 & Piper Rudnick & Etats-Unis - National & 799 & $0 \%$ & 1 \\
\hline 35 & Freehills & Australie - National & 796 & $3 \%$ & 3 \\
\hline 36 & Hogan \& Hartson & $\begin{array}{l}\text { Etats-Unis - } \\
\text { Washington D.C. }\end{array}$ & 793 & $12 \%$ & 11 \\
\hline 37 & Greenberg Traurig & Etats-Unis - National & 788 & $0 \%$ & 1 \\
\hline 38 & O'Melveny \& Myers & $\begin{array}{l}\text { Etats-Unis - Los } \\
\text { Angeles }\end{array}$ & 776 & $4 \%$ & 4 \\
\hline 39 & Simmons \& Simmons & $\begin{array}{l}\text { Royaume-Uni- } \\
\text { Londres }\end{array}$ & 769 & $38 \%$ & 11 \\
\hline 40 & Hunton \& Williams & $\begin{array}{l}\text { Etats-Unis - } \\
\text { Richmond }\end{array}$ & 766 & $5 \%$ & 5 \\
\hline 41 & Fulbright \& Jaworski & Etats-Unis - Houston & 762 & $1 \%$ & 4 \\
\hline 42 & $\begin{array}{l}\text { Squire, Sanders \& } \\
\text { Dempsey }\end{array}$ & Etats-Unis - National & 760 & $22 \%$ & 13 \\
\hline 43 & Clayton Utz & Australie - National & 759 & $0 \%$ & 1 \\
\hline 44 & $\begin{array}{l}\text { Paul, Hastings, Janofsky \& } \\
\text { Walker }\end{array}$ & Etats-Unis - National & 751 & $13 \%$ & 4 \\
\hline 45 & Kirkland \& Ellis & Etats-Unis - Chicago & 747 & $4 \%$ & 2 \\
\hline 46 & Dechert & Etats-Unis - National & 744 & $28 \%$ & 5 \\
\hline 47 & McCarthy Tetrault & Canada - National & 740 & $2 \%$ & 3 \\
\hline 48 & Gowlinig Lafleur Henderson & Canada - National & 727 & $1 \%$ & 2 \\
\hline 49 & $\begin{array}{l}\text { LeBoeuf, Lamb, Greene \& } \\
\text { MacRae }\end{array}$ & Etats-Unis - National & 722 & $15 \%$ & 10 \\
\hline 50 & Blake Dawson Waldron & Australie - National & 716 & $2 \%$ & 4 \\
\hline
\end{tabular}

Source : The American Lawyer/Legal Business 


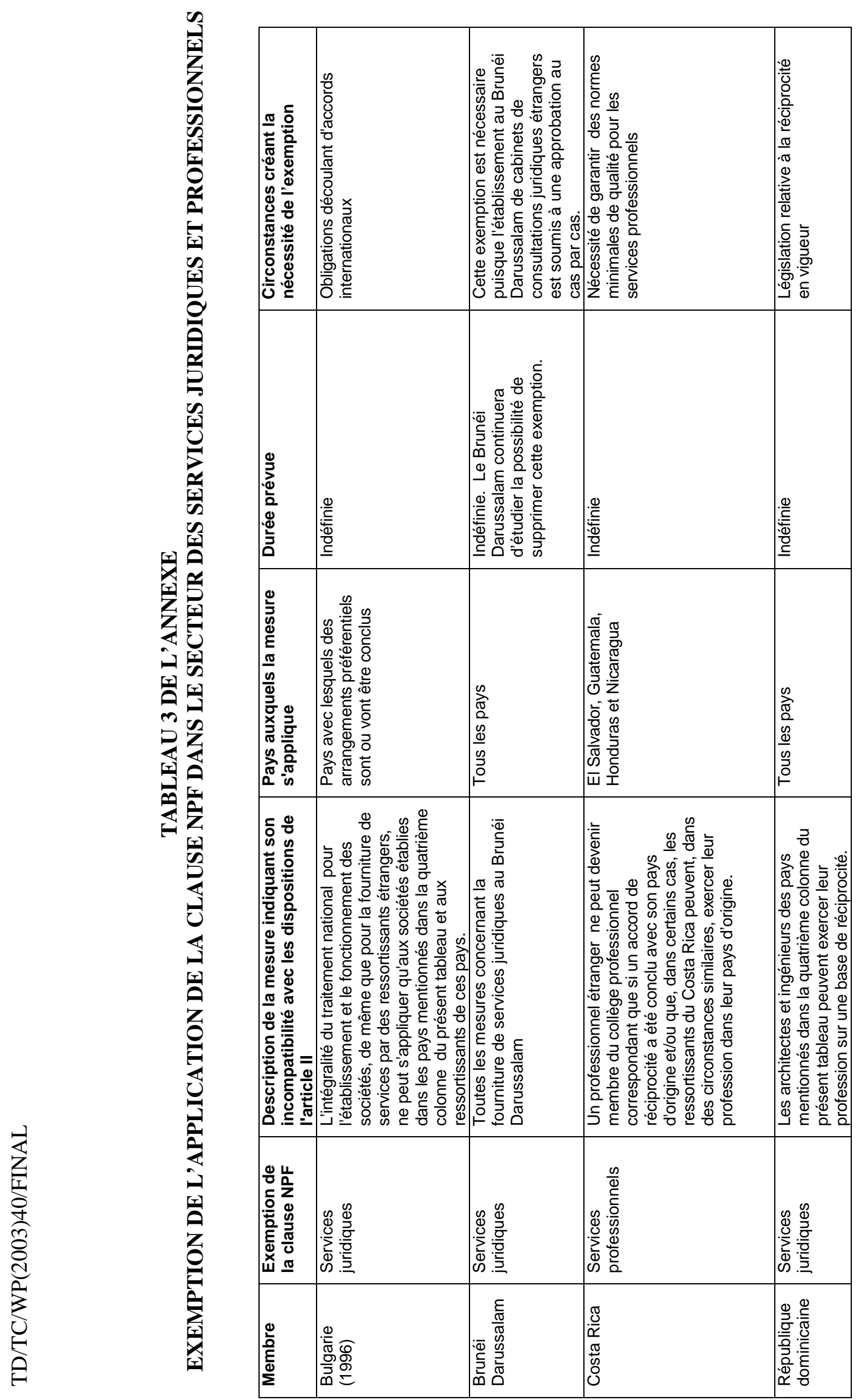




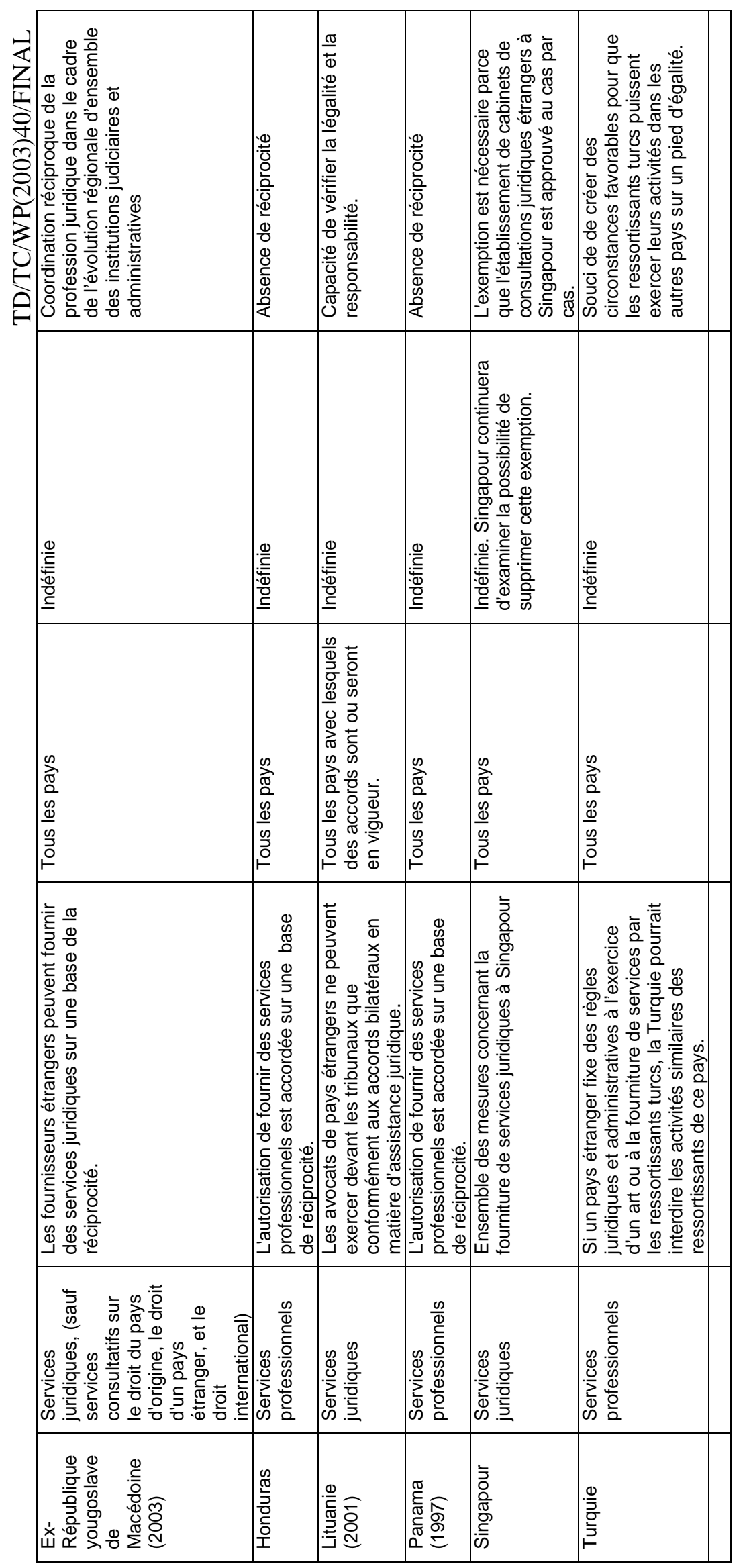

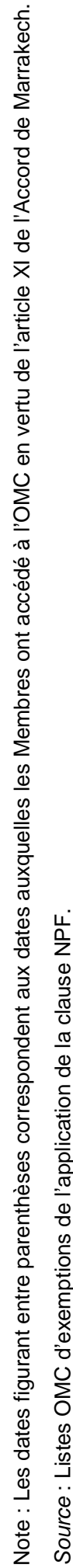




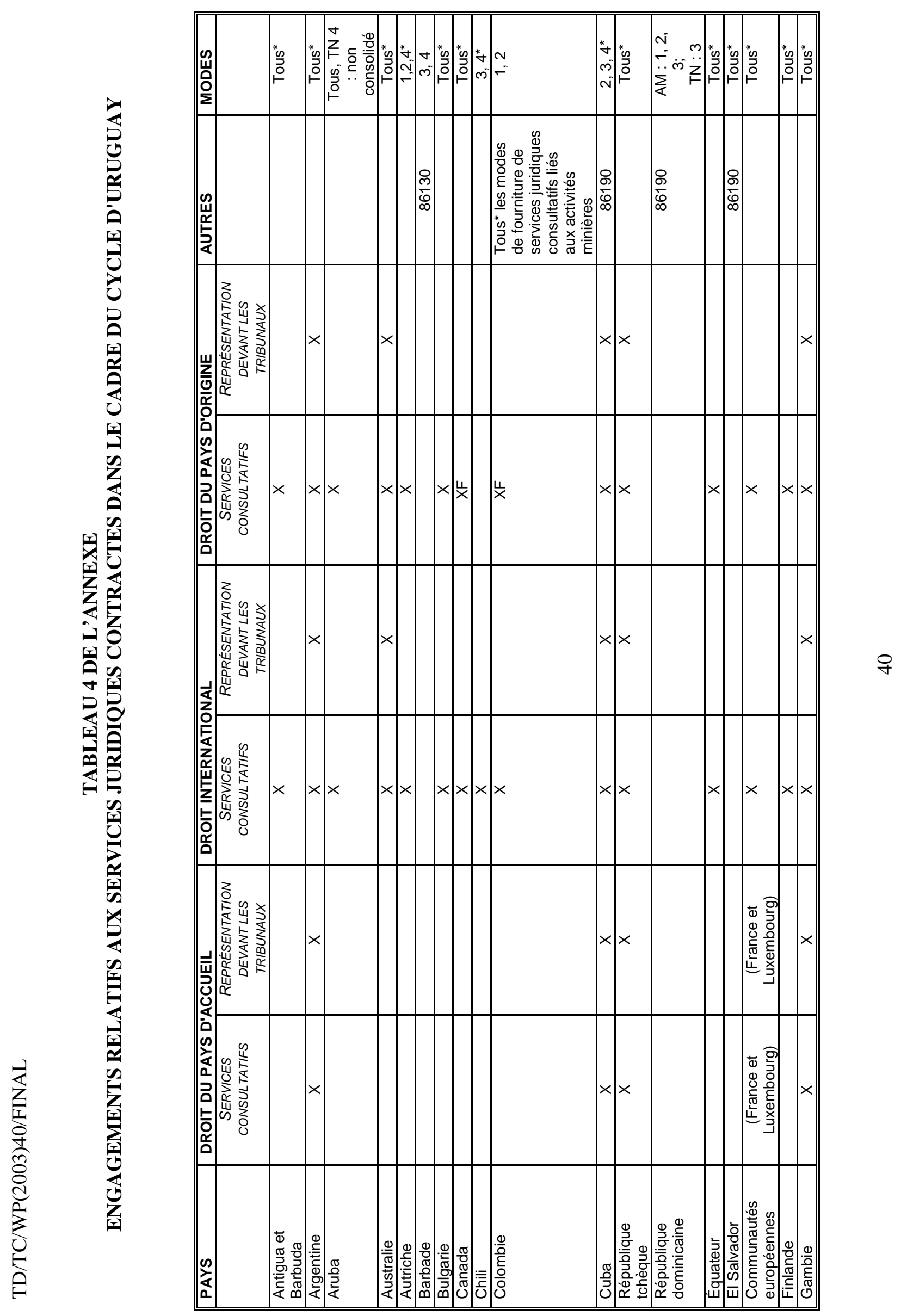




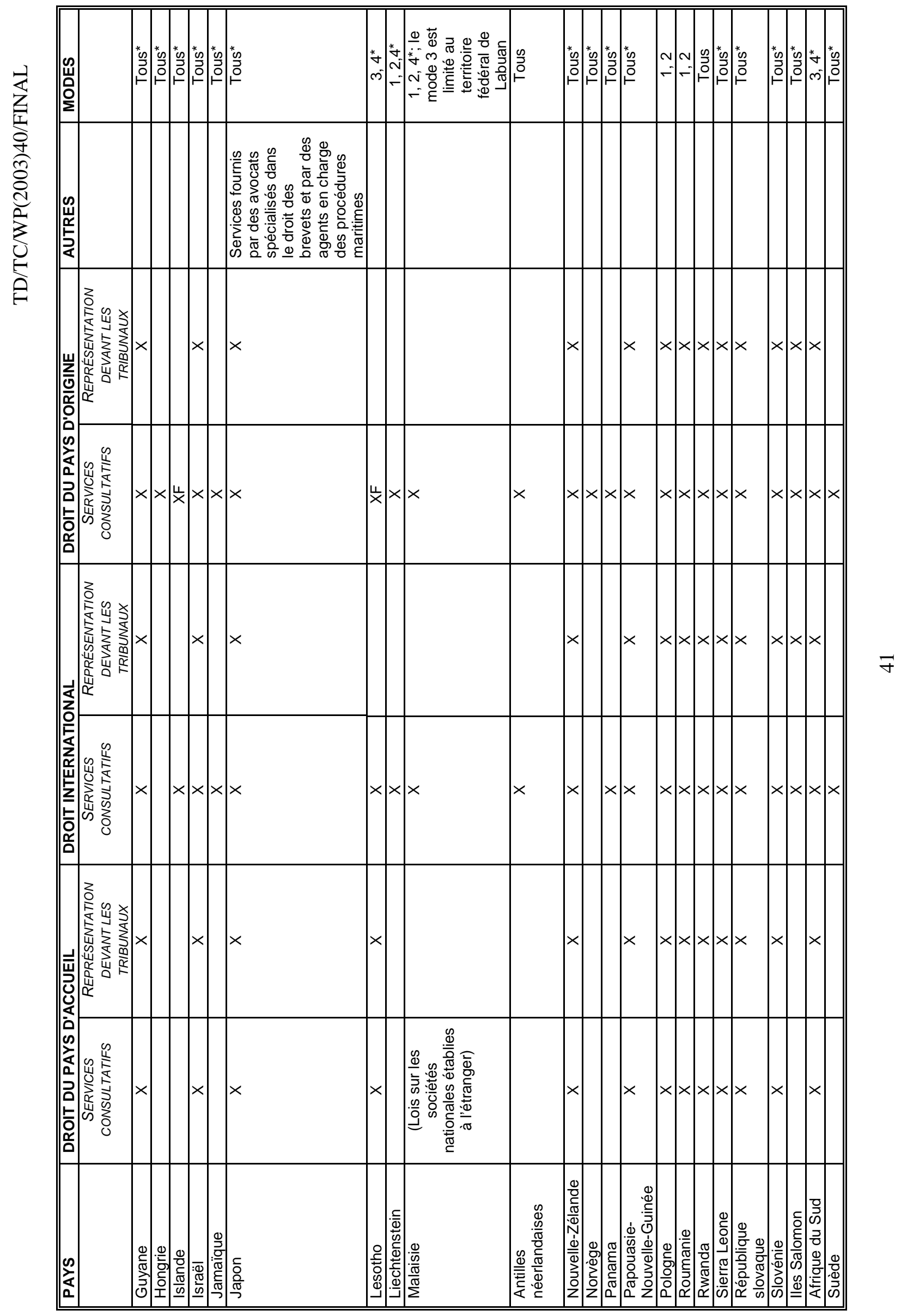




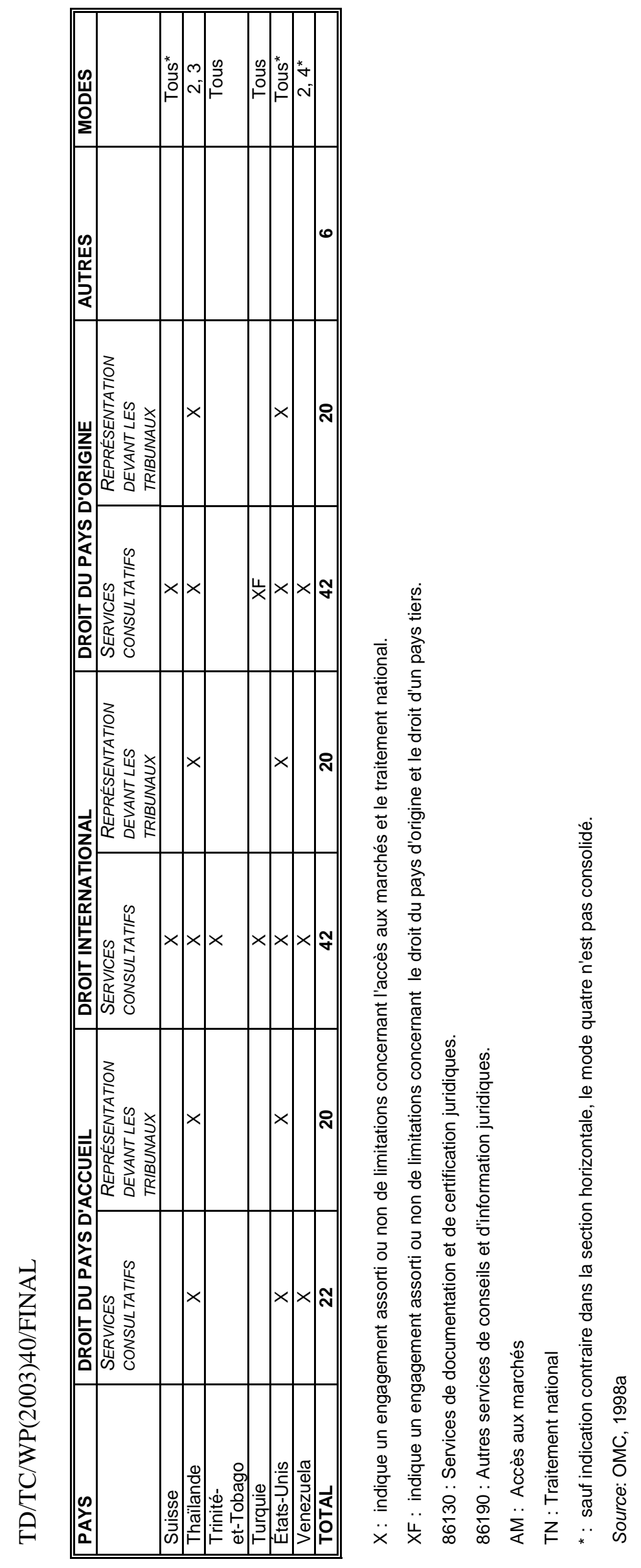




\section{RÉFÉRENCES}

Baker \& McKenzie (2003), « Firm Profile». Disponible sur le site :

www.bakernet.com/BakerNet/Firm+Profile/default.htm

CNUCED (2003), «Organisation des négociations fondées sur le principe des 'offres et requêtes' dans le cadre de l'AGCS : les services du secteur de l'énergie », Genève.

Commission européenne (2002), «Proposition de Directive du Parlement européen et du Conseil relative à la reconnaissance des qualifications professionnelles », $\operatorname{COM}(2002) 119,2002 / 0061$ (COD), en date du 07.03.2002.

Cone, M. S. (2003), « Legal Services in the Doha Round », Journal of World Trade 37 (1): 29-47.

Department for Constitutional Affairs, UK (2002), «WTO and Legal Services: Speech to Delegates”, Rosie Winterton, Parliamentary Secretary, Department for Constitutional Affairs, International Symposium on WTO and Legal Services, Beijing, Chine, septembre 2002.

Department for Constitutional Affairs, UK (2003a), «The Doha Round and Trade in Legal Services » Baroness Scotland of Asthal QC, Parliamentary Secretary, Department for Constitutional Affairs, British Chamber of Commerce in Belgium, Bruxelles, 18 mars 2003.

Department for Constitutional Affairs, UK (2003b), «Law without Frontiers: Legal Services at the Heart of World Trade», Baroness Scotland of Asthal QC, Parliamentary Secretary, Department for Constitutional Affairs, WTO \& Legal Services Conference, Londres, 14 février 2003.

Eurostat (2002), «Enterprises Européennes : Faits et chiffres », données 1990-2000. Communautés européennes.

Hguyen-Hong, D. (2000), «Restrictions on Trade in Professional Services », Productivity Commission Staff Research Paper, AusInfo, Canberra, août.

Hook (2003), « Liberalisation without Harmonisation: The Role of Domestic Regulation », Law Society of England and Wales.

IBA (1998), «Statement of General Principles for the Establishment and Regulation of Foreign Lawyers ».

IBA (2003a), «Resolution in Support of a system of Terminology for Legal Services for the Purposes of International Trade Negociations ». Adopted at the IBA Council Meeting, San Francisco, septembre 2003.

IBA (2003b), «What Changes are Required to the WTO Accountancy Disciplines before they can be applied to the Legal Profession », IBA WTO Working Group Discussion Paper. 
International Financial Services London (2003), «Legal Services », City Business Series 2003.

Johnston, B. (2002), «International Trade in Legal Services: Opportunities and Threats », Communication présentée à la $24^{\mathrm{e}}$ Conférence sur le droit commercial international, Canberra, Australie, 9 octobre 2002.

Lowtax.net (2003), «Hong Kong Professional Services», Disponible sur le site www.lowtax.net/lowtax/html/hongkong/jhprof.html.

Miller, R., et S. Gallacher (2002), « The GATS Framework and Trade in Professional (Legal) Services », Communication présentée à la $24^{\mathrm{e}}$ Conférence sur le droit commercial international, 9 octobre 2002, Canberra, Australie.

New York State Bar Association (2000), «Preserving the Core values of the American Legal Profession: The Place of Multidisciplinary Practice in the Law Governing Lawyers », Report of the New York State Bar Association Special Committee on the Law Governing Firm Structure and Operation. Disponible sur le site : www.law.cornell.edu/ethics/mdp1.htm.

OCDE (1996), International Trade in Professional Services: Assessing Barriers and Encouraging Reform, Paris.

OCDE (1997), International Trade in Professional Services: Advancing Liberalisation through Regulatory Reform, Paris.

OCDE (2002a), «Organisation des négociations fondées sur le principe des 'offres et requêtes' dans le cadre de l'AGCS ». Document TD/TC/WP(2002)13/FINAL. Paris.

OCDE (2002b), «Pour l'ouverture des marchés de services: l'Accord général sur le commerce des services », Paris.

OCDE (2002c), «Circulation des prestataires de services : Accords de reconnaissance mutuelle », Document TD/TC/WP(2002)48/FINAL. Paris.

OCDE (2003a), «Organisation des négociations fondées sur le principe des 'offres et requêtes' dans le cadre de l'AGCS : les services d'assurance », Document TD/TC/WP(2003)17/FINAL. Paris.

OCDE (2003b), «L'investissement direct étranger dans les services professionnels : renforcer la transparence des réglementations nationales». Perspectives de l'investissement international, Edition 2003, Paris.

OMC (1998a), « Services juridiques - Note d'information du Secrétariat », Document S/C/W/43.

OMC (1998b), «Communication des États-Unis - Services juridiques », Document S/C/W/80.

OMC (1999), «Communication de l'Australie », Document S/WPDR/W/1.

OMC (2000a), «Communication de l'Inde : Libéralisation proposée du mouvement des professionnels au titre de l'Accord général sur le commerce des services (AGCS) », Document S/CSS/W12.

OMC (2000b), « Communication des États-Unis - Services juridiques », Document S/CSS/W/28. 
OMC (2000c), «Communication des Communautés européennes et de leurs États membres AGCS 2000 : Services professionnels », Document S/CSS/W/33.

OMC (2000d), «Communication des Communautés européennes et de leurs États membres - Applicabilité des disciplines relatives à la réglementation intérieure dans le secteur des disciplines comptables à d'autres services professionnels », Document S/WPDR/W/5.

OMC (2000e), «Communication de Hong Kong, Chine : Disciplines relatives à la réglementation intérieure pour les services professionnels - Résultat de la consultation avec les secteurs professionnels », Document S/WPDR/W/3.

OMC (2000f), «Communication du Japon - Compte rendu des résultats de l'enquête sur les services professionnels », Document S/WPDR/W/6.

OMC (2000g), «Communication de la République de Corée - Disciplines relatives à la réglementation intérieure pour les services professionnels - Résultats de la consultation avec les secteurs professionnels », Document S/WPDR/W/10.

OMC (2000h), «Communication de la Pologne - Disciplines concernant la réglementation intérieure et les services professionnels - Résultats de la consultation des secteurs professionnels », Document S/WPDR/W/7.

OMC (2000i), «Communication de l'Australie : Nécessité et transparence », Document S/WPDR/W/8.

OMC (2000j), «Communication des États-Unis - Article VI:4 de l'AGCS : Disciplines éventuelles concernant la transparence de la réglementation intérieure », Document S/WPDR/W/4.

OMC (2001a), «Communication de l'Australie : Proposition de négociation pour les services juridiques », Document S/CSS/W/67.

OMC (2001b), «Communication du Canada - Proposition initiale en vue des négociations pour les services professionnels », Document S/CSS/W/52.

OMC (2001c), «Communication de l'Australie : Proposition de négociation pour les services juridiques Révision », Document S/CSS/W/67/Suppl.1/Rev.1.

OMC (2001d), « Communication de la Colombie - Services professionnels », Document S/CSS/W/98.

OMC (2001e), «Communication de la Suisse - AGCS 2000 : Services professionnels », Document $\mathrm{S} / \mathrm{CSS} / \mathrm{W} / 75$.

OMC (2001f), «Communication du Canada - Disciplines relatives à la réglementation intérieure pour les services professionnels - Résultats des consultations avec les secteurs professionnels ». Document S/WPDR/W/13.

OMC (2001g), « Communication du Mexique - Disciplines relatives à la réglementation intérieure pour les services professionnels - Résultats des consultations avec les secteurs professionnels ». Document S/WPDR/W/12.

OMC (2001h), «Communication de la Suisse - Applicabilité des disciplines relatives à la réglementation intérieure dans le secteur des disciplines comptables à d'autres services professionnels ». Document S/WPDR/W/16. 
OMC (2001i), «Communication de la Thaïlande - Résultats de la consultation sur l'applicabilité des disciplines relatives aux services comptables aux autres professions ». Document S/WPDR/W/18.

OMC (2001j), «Communication des Communautés européennes et de leurs États membres Réglementation intérieure : Nécessité et transparence ». Document S/WPDR/W/14.

OMC (2002a), «Communication de l'Australie, Proposition de négociation : Classification des services juridiques - Supplément ». Document S/CSS/W/67Suppl.2.

OMC (2002b), «Communication de la République populaire de Chine - Disciplines relatives à la réglementation intérieure pour les services professionnels - Résultats des consultations avec les secteurs professionnels ». Document S/WPDR/W20.

OMC (2003a), «Communication des Communautés européennes - Classification des services juridiques ». Document S/CSC/W/39.

OMC (2003b), «Communication du Canada - Disciplines relatives à la réglementation intérieure pour les services professionnels - Résultats des consultations avec les secteurs professionnels ». Document S/WPDR/W/22.

OMC (2003c), «Communication du Territoire douanier distinct de Taiwan, Panghu, Kinmen et Matsu Disciplines relatives à la réglementation intérieure pour les services professionnels - Résultats des consultations avec les secteurs professionnels ». Document S/WPDR/W/21.

OMC (2003d), «Communication de la Communauté européenne et de ses États membres - Proposition concernant des disciplines relatives aux procédures de licences ». Document S/WPDR/W/25.

Silver, C. (à paraître), «Regulatory Mismatch in the International Market for Legal Services ». Northwestern Journal of International Law \& Business. Northwestern University School of Law, Chicago, États-Unis.

Singapore Ministry of Law (2003), «Census of Law Firms and Lawyers in Singapore". Communiqué de presse. Disponible sur le site http://app.sprinter.gov.sg/data/pr/2003031302.htm.

Tdctrade.com (2003), «Profiles of Hong Kong Major Service Industries: Legal». www.tdctrade.com/main/si/splega.htm.

Terry, L.S. (2001), “'GATS' Applicability to Transnational Lawyering and its Potential Impact on US State Regulation of Lawyers ». 34 Vanderbilt Journal of Transnational Law 989.

UK Government (2003), « Government Promotes Liberalisation of Legal Services in Korea, Malaysia and Philippines ». Disponible sur le site : http://www.gnn.gov.uk/gnn/national.nsf/0/FC57C76A1C2A70A280256DB40054B600?opendocume nt

UK Office of National Statistics (2003), « United Kingdom balance of Payments: The Pink Book 2003 ». Editor: Simon Linden. 